\title{
MIT
}

\section{Systemically Administered Hemostatic Nanoparticles for Identification and Treatment of Internal Bleeding}

The MIT Faculty has made this article openly available. Please share how this access benefits you. Your story matters.

\begin{tabular}{|l|l|}
\hline As Published & $10.1021 /$ acsbiomaterials.9b00054 \\
\hline Publisher & American Chemical Society (ACS) \\
\hline \hline Version & Author's final manuscript \\
\hline Citable link & https://hdl.handle.net/1721.1/136183 \\
\hline Terms of Use & $\begin{array}{l}\text { Article is made available in accordance with the publisher's } \\
\text { policy and may be subject to US copyright law. Please refer to the } \\
\text { publisher's site for terms of use. }\end{array}$ \\
\hline
\end{tabular}




\section{Systemically Administered Hemostatic Nanoparticles for Identification and Treatment of Internal Bleeding}

Manos Gkikas, ${ }^{\S, \perp, \dagger}$ Thomas Peponis, ${ }^{\ddagger}$ Tomaz Mesar, ${ }^{\ddagger}$ Celestine Hong, ${ }^{\S, \perp}$ Reginald K. Avery, ${ }^{\nabla, \perp}$ Emmanuel Roussakis, ${ }^{\text {I }}$ Hyung-Jin Yoo, ${ }^{\bigcirc}$ Anushri Parakh, ${ }^{\nabla}$ Manuel Patino, $\nabla$ Dushyant V. Sahani, ${ }^{\nabla}$ Michael T. Watkins, ${ }^{\circ}$ Rahmi Oklu, "Conor L. Evans, "I Hassan Albadawi, " George Velmahos, *, and Bradley D. Olsen $*, \S, \perp_{\mathbb{C}}$

${ }^{\S}$ Department of Chemical Engineering, Massachusetts Institute of Technology, Cambridge, Massachusetts 02139, United States

${ }^{\perp}$ Institute for Soldier Nanotechnologies, Massachusetts Institute of Technology, Cambridge, Massachusetts 02139, United States

${ }^{\dagger}$ Department of Chemistry, University of Massachusetts Lowell, Lowell, Massachusetts 01854, United States

${ }^{\ddagger}$ Department of Trauma, Emergency Surgery and Surgical Critical Care, Massachusetts General Hospital, Harvard Medical School, Boston, Massachusetts 02144, United States

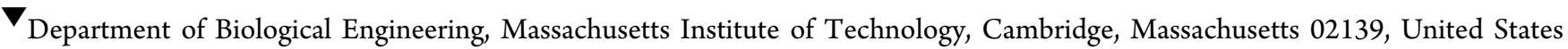

II Wellman Center for Photomedicine, Massachusetts General Hospital, Charlestown, Massachusetts 02129, United States

${ }^{\circ}$ Division of Vascular and Endovascular Surgery, Massachusetts General Hospital, Boston, Massachusetts 02144, United States

${ }^{\nabla}$ Department of Radiology, Massachusetts General Hospital, Boston, Massachusetts 02144, United States

"Division of Vascular and Interventional Radiology, Mayo Clinic, Scottsdale, Arizona 85259, United States

Supporting Information

ABSTRACT: Internal bleeding is an injury that can be difficult to localize and effectively treat without invasive surgeries. Injectable polymeric nanoparticles have been developed that can reduce clotting times and blood loss, but they have yet to incorporate sufficient diagnostic capabilities to assist in identifying bleeding sources. Herein, polymeric nanoparticles were developed to simultaneously treat internal bleeding while incorporating tracers for visualization of the nanoparticles by standard clinical imaging modalities. Addition of $1,1^{\prime}$-dioctadecyl-3,3,3', $3^{\prime}$ tetramethylindodicarbocyanine perchlorate ( $\mathrm{DiD}$; a fluorescent dye), biotin functionality, and gold nanoparticles to hemostatic polymeric nanoparticles resulted in nanoparticles amenable to imaging with near-infrared (NIR) imaging, immunohistochemistry, and X-ray computed tomography (CT), respectively. Following a lethal liver resection injury, visualization of accumulated nanoparticles by multiple imaging methods was achieved in rodents, with the highest accumulation observed at the liver injury site, resulting in improved survival rates. Tracer addition to therapeutic nanoparticles allows for an expansion of their applicability, during stabilization by first responders to diagnosis and identification of unknown internal bleeding sites by clinicians using standard clinical imaging modalities.

KEYWORDS: hemostasis, imaging, theranostic, bleeding, internal injuries, injectable, nanoparticle

\section{INTRODUCTION}

Localizing a source of internal bleeding can improve diagnostic and therapeutic efficacy in the field or in a clinical environment. ${ }^{1,2}$ Internal bleeding sources, for both traumatic injuries and chronic conditions, are difficult to identify without an invasive procedure. ${ }^{3-6}$ Minimally invasive techniques have reduced patient discomfort for some procedures associated with identifying internal bleeding sources and can also be used to simultaneously deliver therapeutic materials. ${ }^{7-10}$ Theranostic treatments combine diagnostic abilities with a therapy into one system, resulting in improved treatment efficacy and reduced procedural time for patients. Cancer treatments take advantage of this approach when using small molecules, proteins, or particles to target cancerous tissue and locally deliver a therapy, reducing the toxic systemic effects of many cancer drugs. ${ }^{11}$ Similarly, internal bleeding could benefit from treatments that can diagnose sources of bleeding while effectively treating bleeding. With these systems, bleeding times and blood loss can be minimized while the source of bleeding is identified to better assess the need for further treatment.

The potential of theranostic approaches to address internal bleeding could benefit first responders and clinicians during patient care. Patients exhibiting external signs of internal bleeding can be stabilized on site, ${ }^{6}$ or a bleeding diagnosis can be expedited by clinicians in a hospital. ${ }^{12,13}$ Initial delivery of a theranostic material could achieve patient stabilization prior to their presence at a hospital. Even at a hospital, there are

Received: January 11, 2019

Accepted: February 25, 2019 
bleeding conditions that can be difficult to localize with a computed tomography (CT) scan or angiography. Intermittent gastrointestinal bleeding can be difficult to identify by imaging of injected contrast dyes due to their irregular bleeding frequency. ${ }^{14}$ The presence of a constant signal from a bleeding site would serve as a guide for both invasive and minimally invasive treatments. Moreover, with the introduction of mobile CT scanners, ${ }^{15}$ theranostic nanoparticles (NPs) could lead to preliminary diagnoses that could expedite treatment in transit or upon arrival at the hospital.

Unfortunately, there are only a few examples of theranostic solutions for bleeding or clotting conditions. One example of a bleeding or clot-related theranostic is a microbubble designed for clot identification and subsequent clot lysis. ${ }^{16,17}$ Plaque buildup in vessels can result in the generation of a clot, which can result in a stroke or myocardial infarct. Antibodies and fibrinolytic drugs were combined on the surface of a microbubble to target activated platelets and lyse clots, respectively. The microbubbles were capable of enhanced imaging by ultrasound and clots were shown to be identified and treated in cases of myocardial infarction in mice. The fibrinolytic drug, known to be efficacious but harmful when systematically delivered, was localized in its effects and targeted in its activity, resulting in a high, localized concentration of drug that reduced systemic bleeding side effects in vivo. Another example involves the use of fibrin-specific probes to detect thrombus location. ${ }^{16}$ By using a peptide with $>100$-fold specificity for fibrin over fibrinogen, this was found to result in high probe uptake by the thrombotic artery. Although this particular target relates closely to the maintenance of hemostasis, there has yet to be a study that employs a variety of labeling methods in a theranostic specifically to treat internal bleeding.

To develop more theranostic approaches for bleeding conditions, the available clinical imaging modalities must be considered during system design. One of the more common imaging modalities for bleeding and clotting visualization, in addition to ultrasound-based imaging, ${ }^{17,18}$ is computed tomography (CT) angiography. CT angiography commonly uses heavy element contrast dyes, commonly including iodine, in particles or solutions to visualize a bleeding location by $\mathrm{X}$ ray imaging. ${ }^{19}$ Other studies in animals involve histology and immunohistochemistry or imaging with near-infrared (NIR) dyes for more topical sources of bleeding in real-time or postsacrifice. $^{20,21}$ Magnetic resonance imaging (MRI) is also used to identify aged blood clots with molecules responsive to $\mathrm{pH}$, blood oxygen levels, and other metrics that characterize blood clots in vivo. ${ }^{22}$ Positron emission tomography (PET) has also been used by attaching radiolabeled markers to peptides or other materials. ${ }^{23}$ This can allow for a single full body scan to identify any sources of bleeding, though the expense of the procedure and additional requirements, relative to X-ray or ultrasound, can limit its use in emergency situations. Despite the variety of imaging modalities that can be used to visualize bleeding or clotting locations, theranostic systems utilizing these imaging modalities have not been well developed.

The particles that have been developed for managing internal bleeding are capable of being utilized for both diagnostic and therapeutic treatments, though therapeutic treatments for nonlethal bleeding models represent the majority of reported studies. Liposomes ${ }^{24-26}$ and polymeric nanoparticles ${ }^{27-31}$ have been designed with functional groups exposed to interact with matrix proteins or cell receptors uniquely present at wound sites. Platelet receptors, collagen, von Willebrand factor (vWF), and fibrinogen sequences are all examples of sites targeted by hemostatic particles. ${ }^{26,27}$ The Gupta group has investigated adhesive peptides such as von Willebrand factor-binding peptides (VBP) and the therapeutic effects of combining aggregating and adhesive moieties on liposomes in a tail transection injury model, ${ }^{32}$ while Okamura et al. focused on a fibrinogen-derived dodecapeptide (H12) on albumin microparticles. ${ }^{33}$ The Lavik group has shown the potential of poly(lactic-co-glycolic acid)- $b$-poly(L-lysine)- $b$ poly(ethylene glycol) (PLGA- $b$-PLL- $b$-PEG) particles to manage internal bleeding. ${ }^{27}$ Functionalized with RGD, these nanoparticles have shown the ability to reduce clotting times and blood loss in liver bleeding as well as blunt and blast trauma models. ${ }^{28-31,34}$ Further investigations surrounding the impact of particle thermal stability ${ }^{29}$ and ligand density ${ }^{30}$ on clotting have been performed to improve their performance in rodent and porcine bleeding models. Steroid-loaded nanoparticles were also shown to encourage accelerated healing and prevent damage caused by inflammation, improving the therapeutic performance of the particles. ${ }^{34}$

In this work, we endeavor to include a diagnostic functionality to these therapeutically active nanoparticles and assess their performance with a lethal injury model. By inclusion of dyes and X-ray contrast agents, the nanoparticle's capability to be employed as a diagnostic tool was showcased. Perturbations in coagulation and platelet aggregation extent upon addition of nanoparticles were assessed in vitro. In vivo, the particles containing diagnostic markers were shown to maintain the therapeutic performance of unlabeled particles, suggesting that the presence of dyes or contrast agents did not inhibit or alter the therapeutic performance of the particles. Biodistribution trends were varied depending on the marker and imaging technique used to quantify particle concentrations in each organ, possibly due to differences in particle size and variable stability of the markers within the particles after delivery. Overall, our engineered PLGA-PEG nanoparticles have the potential to be utilized as theranostic particles, providing diagnostic capabilities and therapeutic performance for lethal injuries.

\section{MATERIALS AND METHODS}

Materials. Poly(ethylene glycol) bis(amine) $\left(M_{\mathrm{w}} 6000\right)$, acidterminated poly(D,L-lactide-co-glycolide) (PLGA: lactide/glycolide 75:25, $M_{\mathrm{w}}$ 4000-15000), 4-dimethylaminopyridine (DMAP), $N, N^{\prime}$ dicyclohexylcarbodiimide (DCC), and 1,1'-carbonyldiimidazole (CDI) were purchased from Sigma-Aldrich. GRGDS peptide (95\%) was purchased from China Peptides. Amine- $\mathrm{PEG}_{2}$-biotin, Alexa Fluor 350 streptavidin, horseradish peroxidase (HRP)-conjugated streptavidin, and the NIR-fluorescent dye 1,1'-dioctadecyl-3,3,3',3'-tetramethylindodicarbocyanine perchlorate $(\mathrm{DiD})$ were purchased from Thermo Fisher Scientific. Tyramide signal amplification (TSA) solution reagent kit containing cyanine 3 was purchased from PerkinElmer. Dodecanethiol functionalized gold nanoparticles $(\sim 5$ $\mathrm{nm}$ in diameter) were purchased from Nanoprobes. Deuterated solvents $\left(\mathrm{CDCl}_{3}\right.$ and DMSO- $\left.d_{6}\right)$ were purchased from Cambridge Isotope Laboratories. Citrated whole human blood was acquired from Research Blood Components (Brighton, MA). Both male and female Sprague-Dawley rats $(300-400 \mathrm{~g})$ were purchased from Charles River Laboratories.

Synthesis of Poly(D,L-lactide-co-glycolide)-b-Poly(ethylene oxide)-amine (PLGA-b-PEG-NH $\left.{ }_{2}\right)$. PLGA-COOH $\left(2 \mathrm{~g}, \sim 2 \times 10^{-4}\right.$ $\mathrm{mol}, \sim 10000 \mathrm{~g} / \mathrm{mol})$, DMAP $\left(9.8 \mathrm{mg}, 0.8 \times 10^{-4} \mathrm{~mol}\right.$, 0.4 equiv $)$, and $\mathrm{H}_{2} \mathrm{~N}-\mathrm{PEG}-\mathrm{NH}_{2}\left(1.2 \mathrm{~g}, 2 \times 10^{-4} \mathrm{~mol}, 6000 \mathrm{~g} / \mathrm{mol}\right)$ were dissolved in 12 wt $\%$ DCM $(16.7 \mathrm{~mL})$. Finally, DCC $\left(62 \mathrm{mg}, 3 \times 10^{-4} \mathrm{~mol}\right.$, 
$206.33 \mathrm{~g} / \mathrm{mol}, 1.5$ equiv) was added dropwise, and the mixture was stirred at room temperature (RT). The block copolymer was finally precipitated in 1:1 ether/MeOH multiple times, resulting in a final purified yield of $1.5 \mathrm{~g}(75 \%)$.

Synthesis of Poly(D,L-lactide-co-glycolide)-b-Poly(ethylene oxide)-carbonylimidazole (PLGA-b-PEG-carbonylimidazole). PLGA- $b$-PEG-NH ${ }_{2}\left(1.5 \mathrm{~g}, 0.94 \times 10^{-4} \mathrm{~mol}, \sim 16000 \mathrm{~g} / \mathrm{mol}\right)$ was dissolved in $12 \mathrm{wt} \%$ dioxane $(12.5 \mathrm{~mL})$ at $37{ }^{\circ} \mathrm{C}$, and a $6: 1 \mathrm{CDI} /$ PEG molar excess was added $\left(91.3 \mathrm{mg}, 5.63 \times 10^{-4} \mathrm{~mol}, 162.15 \mathrm{~g} /\right.$ $\mathrm{mol}$ ). The resulting mixture was stirred at $37{ }^{\circ} \mathrm{C}$ for $2 \mathrm{~h}$. Ether was added to the reaction mixture to precipitate the product, resulting in a final purified yield of $1.45 \mathrm{~g}$. The resulting activated polymer was stored at $-20{ }^{\circ} \mathrm{C}$.

Synthesis of Poly(D,L-lactide-co-glycolide)-b-Poly(ethylene oxide)-GRGDS (PLGA- $b$-PEG-GRGDS). PLGA- $b$-PEG-carbonylimidazole $\left(0.4 \mathrm{~g}, 0.25 \times 10^{-4} \mathrm{~mol}, \sim 16000 \mathrm{~g} / \mathrm{mol}\right)$ was mixed with the GRGDS peptide $\left(36 \mathrm{mg}, 0.75 \times 10^{-4} \mathrm{~mol}, 489 \mathrm{~g} / \mathrm{mol}, 3\right.$ equiv $)$ in 9 wt \% dry DMSO (4.4 mL) and stirred overnight. Then, the polymer solution was diluted with more DMSO, dialyzed (molecular weight cutoff (MWCO) $3500 \mathrm{~g} / \mathrm{mol}$ ) against deionized water for 3 days to remove unconjugated peptide, and lyophilized to give $0.38 \mathrm{~g}$ of a white solid. To prepare the injectable nanoparticles, the functionalized polymer was dissolved in DMSO and dialyzed (MWCO $3500 \mathrm{~g} / \mathrm{mol}$ ) against deionized water for 3 days to obtain a polymeric particle formulation. Water was then removed after centrifugation for $12 \mathrm{~min}$ at $4700 \mathrm{~g}$, followed by addition of PBS to the resulting pellet, vortexing, and sonication in order to break larger particles. After one more round of centrifugation, PBS was added to achieve a final concentration of $20 \mathrm{mg} / \mathrm{mL}$.

Synthesis of Poly(D,L-lactide-co-glycolide)-b-Poly(ethylene oxide)-biotin (PLGA-b-PEG-biotin). PLGA- $b$-PEG-carbonylimidazole $\left(0.4 \mathrm{~g}, 0.25 \times 10^{-4} \mathrm{~mol}, \sim 16000 \mathrm{~g} / \mathrm{mol}\right)$ was mixed with amine$\mathrm{PEG}_{2}$-biotin $\left(28 \mathrm{mg}, 0.75 \times 10^{-4} \mathrm{~mol}, 374.5 \mathrm{~g} / \mathrm{mol}, 3\right.$ equiv) in $9 \mathrm{wt}$ $\%$ dry DMSO $(4.4 \mathrm{~mL})$ and stirred overnight. Then, the polymer solution was diluted with more DMSO, dialyzed (MWCO $3500 \mathrm{~g}$ / $\mathrm{mol}$ ) against deionized water for 3 days, and lyophilized to give $0.38 \mathrm{~g}$ of a white lyophilized solid.

Fluorescently-Labeled PLGA-b-PEG-GRGDS Nanoparticles. PLGA- $b$-PEG-GRGDS (100 mg) was dissolved in dry DMSO, and $62.5 \mu \mathrm{L} \mathrm{DiD}$ dye (obtained from a $1.46 \mathrm{mM}$ DMSO stock solution or $1.4 \mathrm{mg} / \mathrm{mL})$ or $60 \mathrm{mg}(\sim 0.5 \% \mathrm{wt} / \mathrm{v})$ of coumarin 6 was added. The solution was stirred overnight and then dialyzed (MWCO $3500 \mathrm{~g} /$ $\mathrm{mol}$ ) against deionized water for 3 days. The same procedure used for unloaded PLGA- $b$-PEG-GRGDS nanoparticles was followed without addition of any dye, leading to a final particle concentration of $20 \mathrm{mg} /$ $\mathrm{mL}$.

Gold-Labeled PLGA- $b$-PEG-GRGDS Nanoparticles. PLGA- $b$ PEG-GRGDS (100 mg) was dissolved in dry THF, and $16.67 \mathrm{mg}$ of dodecanethiol-functionalized gold nanoparticles (in dry THF) was added. The solution was stirred overnight and dialyzed (MWCO $3500 \mathrm{~g} / \mathrm{mol}$ ) against deionized water for 3 days. The same procedure used for unloaded PLGA- $b$-PEG-GRGDS nanoparticles was performed, leading to a concentration of $20 \mathrm{mg} / \mathrm{mL}$.

Biotinylated PLGA-b-PEG-GRGDS Nanoparticles. PLGA- $b$ PEG-GRGDS and PLGA- $b$-PEG-biotin (100 $\mathrm{mg}$ of each) were dissolved in DMSO, stirred overnight, and dialyzed (MWCO $3500 \mathrm{~g}$ / $\mathrm{mol}$ ) against deionized water for 3 days. The sample was then centrifuged; the water was removed, and PBS was added. Nanoparticles were then vortexed, forming a homogeneous milky solution, followed by another centrifugation and an additional PBS step, until a final particle concentration of $20 \mathrm{mg} / \mathrm{mL}$ was reached.

Blood Components Preparation. Platelet rich plasma (PRP) for coagulation and aggregation tests was made by centrifuging citrated human whole blood at $200 \mathrm{~g}$ in a $15 \mathrm{~mL}$ conical tube for $10 \mathrm{~min}$. The supernatant was collected and aspirated into a new conical tube. Platelet concentrate (PC) (where all the clotting factors have been removed by multiple centrifugations, leaving only platelets) and platelet poor plasma (PPP) were formed by spinning PRP at $2000 \mathrm{~g}$ for $10 \mathrm{~min}$. PPP (supernatant) was removed, and the pellet was suspended in wash buffer $(134 \mathrm{mM} \mathrm{NaCl}, 3 \mathrm{mM} \mathrm{KCl}, 0.3 \mathrm{mM}$
$\mathrm{NaH}_{2} \mathrm{PO}_{4}, 2 \mathrm{mM} \mathrm{MgCl}$, $5 \mathrm{mM}$ HEPES, $5 \mathrm{mM}$ glucose, $12 \mathrm{mM}$ $\mathrm{NaHCO}_{3}, 1 \mathrm{mM}$ EDTA, $3.5 \mathrm{mg} / \mathrm{mL}$ BSA at $\left.\mathrm{pH}=6.5\right)$. The solution was centrifuged again at $2000 \mathrm{~g}$, and the pellet was suspended in suspension buffer (wash buffer with $0.2 \mathrm{mg} / \mathrm{mL}$ fibrinogen and without EDTA) at a concentration of $3 \times 10^{8}$ platelets $/ \mathrm{mL}$.

Coagulation Measurements. Combinations of $90 \mu \mathrm{L}$ of PRP, 10 $\mu \mathrm{L}$ of $\mathrm{CaCl}_{2}$, and PLGA- $b$-PEG-GRGDS nanoparticles $(7.5 \mu \mathrm{L}$ of 20 $\mathrm{mg} / \mathrm{mL}$ ) or buffer (equal volumes of $0.9 \mathrm{wt} \%$ sodium chloride) were mixed in 96 well plates. All components except PRP were initially added to wells while PRP was simultaneously added to all wells using a multichannel pipet. Well plates were placed in a microplate reader capable of temperature control (set at $37{ }^{\circ} \mathrm{C}$ ), and transmission measurements at $650 \mathrm{~nm}$ were taken every $20 \mathrm{~s}$ over the span of 20 min. Transmission measurements involving gold nanoparticles were also collected at $450 \mathrm{~nm}$ to minimize the effect of absorbance from gold aggregates (which shift to longer wavelengths as particle size increases). The measurements were normalized to positive and negative controls at that specific wavelength. All tests were performed in triplicate.

Platelet Aggregation Measurements. Combinations of $90 \mu \mathrm{L}$ of PRP (or PC), $10 \mu \mathrm{L}$ of agonist (ADP, collagen, or thrombin), and multiple concentrations of nanoparticles $(0.5,1.0,2.0,7.5,10.0$, and $15.0 \mu \mathrm{L}$ at $20 \mathrm{mg} / \mathrm{mL}$ ) were mixed in 96 well plates. Aliquots of 0.1 $\mathrm{mM}$ ADP, $1 \mathrm{mg} / \mathrm{mL}$ collagen in $0.1 \mathrm{M}$ acetic acid, or $100 \mathrm{U} / \mathrm{mL}$ thrombin were used and mixed initially with the hemostat components or buffer to equalize volumes among all the samples. This mixture was then added to PRP or PC, and $100 \mu \mathrm{L}$ (total volume) was aliquoted to each well. Well plates were placed in a microplate reader at $37^{\circ} \mathrm{C}$ and read at $620 \mathrm{~nm}$ every $15 \mathrm{~s}$ for $15 \mathrm{~min}$. Measurements involving gold nanoparticles were likewise collected at $450 \mathrm{~nm}$ due to possible absorbance at the original wavelength, as detailed in the previous paragraph. The plates were shaken in the plate reader in between measurements to prevent precipitation of the platelets and particles.

In Vivo Rat Model of Surgically Induced Liver Injury. Sprague-Dawley rats (300-400 g) were used in accordance with procedures approved by the Animal Care and Use Committee (IACUC) of Massachusetts General Hospital. Animals had ad libitum access to both food and water until the time of the procedure. Twenty-two animals (both males and females) were randomized into 2 different groups (11 rats each). The animals of the control (first) group received two injections of normal saline $(1.5+1 \mathrm{~mL}$ at 0 and 5 min following the injury, respectively), and those of the second (treatment) group received PLGA- $b$-PEG-GRGDS nanoparticles at $20 \mathrm{mg} / \mathrm{mL}(1.5 \mathrm{~mL}$ at time 0$)+$ normal saline $(1 \mathrm{~mL}$ at $5 \mathrm{~min})$. Finally, 9 additional male rats (third group) underwent the procedure receiving treatment with PLGA- $b$-PEG-GRGDS nanoparticles with incorporated markers $(1.5 \mathrm{~mL}$ at time 0$)+$ saline $(1 \mathrm{~mL}$ at $5 \mathrm{~min})$. The purpose of the latter group was mainly to visualize the nanoparticles and perform biodistribution studies. Polymeric nanoparticles containing a hydrophilic (biotin) and two hydrophobic (DiD, Au-NPs) markers were used (3 animals per marker). All treatments were in saline as a vehicle solution. The primary end points of the study included mortality, survival time, and total blood loss.

Following anesthesia with inhaled isoflurane (2\%), a left femoral cut down was performed. After dissection, the left femoral vein was skeletonized and cannulated with a $24 \mathrm{G}$ catheter. The catheter was then flushed with $0.2 \mathrm{~mL}$ of normal saline. Subsequently, a midline laparotomy was performed, and the abdomen was entered sharply. The liver was exposed, and a liver resection was performed. For the resection, a ruler was used to mark the left and right lateral liver lobes at approximately $2.5 \mathrm{~cm}$ from the inferior edge. Subsequently, the lobes were sharply cut with a scalpel blade (No. 10). This was considered as time zero and the start of the observation period (the resected liver was stored and used as a negative control for biodistribution studies). Immediately following the resection, normal saline (group 1) or GRGDS-nanoparticles (groups 2 and 3) were administered intravenously through the femoral vein catheter (time 0 ). The treatment was allowed to circulate for $5 \mathrm{~min}$, at which point a second injection containing only normal saline was administered, and 
the animal was observed for an additional 175 min or until mortality (determined by the cessation of the pulmonary function). The depth of anesthesia was continuously monitored, and the animals experienced no distress throughout the procedure. Animals that were still alive after the $3 \mathrm{~h}$ observation period were euthanized with pentobarbital $(200 \mathrm{mg} / \mathrm{kg}$, intraperitoneally). Subsequently, the abdomen was re-explored, and blood was collected using preweighed gauzes. The remnant liver was then removed from all animals, in order to calculate the extent of liver resection. Additionally, the kidneys, spleen, heart, lungs, and clots were removed from all euthanized animals of the third group, in order to perform biodistribution studies.

Biodistribution Studies of DiD-Labeled Nanoparticles. Following euthanasia, liver, spleen, heart, kidneys, lungs, and blood clots were harvested. A section of each tissue was placed in separate disposable molds filled with optimal cutting temperature (OCT) compound (Tissue-Tek, Sakura-Americas, Torrance, CA) and stored in a sealed bag at $-80{ }^{\circ} \mathrm{C}$, while the remaining tissue of each organ was placed in a 9-well plate and stored at $-80{ }^{\circ} \mathrm{C}$. Resected liver tissues were harvested at the beginning of the experiment and used as negative controls. Tissue sections were generated at $30 \mu \mathrm{m}$ thickness using a cryomicrotome (Leica Biosystems, Buffalo Grove, IL). The fluorescence signal of $\mathrm{DiD}$ was obtained on an Olympus FV1000 inverted confocal microscope with hyperspectral acquisition capabilities, using the $635 \mathrm{~nm}$ laser diode as the excitation source and the manufacturer's recommended filter settings. Fluorescence images were collected over the wavelength range of $640-720 \mathrm{~nm}$ using a water immersion objective (Olympus UPLSAPO 60×, $1.20 \mathrm{NA}$ ). Lambda scans were serially acquired across the same wavelength range, using $4 \mathrm{~nm}$ steps with a full width at half-maximum (fwhm) resolution of $5 \mathrm{~nm}$ per step, with the purpose of extracting fluorescence spectra to specifically identify $\mathrm{DiD}$ emission (group 3) or its absence for the control group. In order to perform instrument spectral function subtraction from the fluorescence spectra, lambda scans were acquired at the cover glass-air interface (away from tissue sections). The spectra thus obtained were subtracted from the fluorescence spectra generated from lambda scans at the tissue sections, resulting in the background-free spectra shown in Figures $3 \mathrm{c}, \mathrm{f}$ and S16. Colored images were generated using ImageJ software (National Institute of Health, USA).

After imaging these sections, the two sections of each organ (the remaining section from cryosectioning and the other section that was initially frozen separately) were combined together into a plastic conical tube and rinsed three times with PBS to remove any remaining blood (which could interfere in the study) and the OCT compound. The organs were then frozen in liquid nitrogen and lyophilized. The entire dried organs were ground with a mortar and pestle, suspended in acetonitrile, and incubated at $37^{\circ} \mathrm{C}$ for $5 \mathrm{~h}$. This step allowed for dissolution of nanoparticles contained in the tissue and liberation of the fluorescent hydrophobic dye into the organic media. Tubes were then centrifuged at $4700 \mathrm{~g}$ for $12 \mathrm{~min}$ to remove solid matter, and the supernatant was collected. Centrifugation was repeated once more after the addition of fresh acetonitrile to remove soluble extracts in the remaining solid. The two supernatant fractions were combined; the solvent was removed by rotary evaporation, and the tissue extracts of each organ were transferred into vials using the same volume of acetonitrile for all organs. Fluorescence detection per whole organ at isovolumetric conditions was measured on a plate reader (Ex. 640/Em. $665 \mathrm{~nm}$ ), and the results were converted to a concentration based on a calibration curve of DiD-loaded PLGA- $b$ PEG-GRGDS nanoparticles at the same volume given in the injection. Results are plotted as biodistribution (\%) of particles injected, relative to the examined organs.

Immunohistochemistry of Biotinylated Nanoparticles. Frozen tissue sections from different organs were generated at $8 \mu \mathrm{m}$ thickness using a cryomicrotome (Leica Biosystems, Buffalo Grove, IL) and hydrated in PBS for $10 \mathrm{~min}$ at RT. Endogenous biotin activity of tissues was blocked using an avidin/biotin blocking reagent (Invitrogen, Cat \#004303, Thermo Fisher Scientific, Waltham, MA) for $10 \mathrm{~min}$, followed by nonspecific protein blocking using TNB buffer (PerkinElmer, Waltham, MA) for $1 \mathrm{~h}$ at RT. Tissue sections were incubated with streptavidin-conjugated horseradish peroxidase in TNB buffer for $30 \mathrm{~min}$ at RT and finally reacted with TSA cyanine3 (Cy3) in amplifying buffer solution (Cat\# NEL704A001 KT, PerkinElmer, Waltham, MA) for 7 min inside a dark chamber at RT. Slides were then covered with hardening type aqueous mounting medium containing the nucleus-stain 4',6-diamidino-2-phenylindole (DAPI) (Vectashield, Cat\#H-1500, Vector Laboratories, Burlingame, $\mathrm{CA})$. The $\mathrm{Cy} 3$ and DAPI fluorescence signals were imaged using an Olympus BX51 fluorescent microscope system equipped with Cy3 and DAPI compatible filters. Colored images were generated using ImageJ software (National Institute of Health, USA).

Biodistribution Studies of Gold-Loaded Nanoparticles. The same organs as reported above were collected and placed in plastic cups for computed tomography (CT) scanning. Control (salineinjected) and three gold-injected animals were scanned on a dualenergy CT scanner with rapid $\mathrm{kV}$ switching technology (Discovery CT 750 HDCT, GE Healthcare, Milwaukee, WI, USA). Dual-energy $\mathrm{CT}$ scans were acquired with the X-ray tube alternating at a voltage of $80 / 140 \mathrm{kVp}$, with $600 \mathrm{~mA}$ tube current, $0.6 \mathrm{~s}$ rotation time, $64 \times$ $0.625 \mathrm{~mm}$ collimation, and $1.375 \mathrm{~mm}$ pitch. The images were interpreted on a vendor-specific workstation ( $\mathrm{ADW}$ version 4.5, GE Healthcare) using the Gemstone Spectral Imaging Software (GSI Viewer). Virtual monochromatic (VMC) images were created at 40 and $80 \mathrm{keV}$ photon energies. Attenuation information (Hounsfield Units: HU) was obtained at the two VMC levels by selecting three separate regions-of-interest (ROI) from each organ image (18-44 $\mathrm{mm}^{2}$ depending on the organ) and measuring attenuation for each animal imaged. A dual energy ratio ( $\mathrm{HU}$ at 40 and $80 \mathrm{keV}$ ) was computed for each organ and served as an in vivo semiquantitative assessment of gold nanoparticle concentration. For the three test animals, the mean of the DE ratio was calculated and compared with the control.

Characterization Methods. Gel permeation chromatography (GPC) was carried out on an Agilent 1260 GPC system with three DMF ResiPore columns (resolution up to $400 \mathrm{kDa}$ ), a Wyatt MiniDAWN TREOS 3-angle static light scattering detector, and a Wyatt Optilab T-rEX refractive index detector. DMF with $0.01 \mathrm{M} \mathrm{LiBr}$ was used as eluent at $1.0 \mathrm{~mL} / \mathrm{min}$ at $70{ }^{\circ} \mathrm{C} .{ }^{1} \mathrm{H}$ NMR spectroscopy (500 $\mathrm{MHz}$ ) was performed using a Varian Inova 502 spectrometer. Aminoacid analysis was performed using an Agilent 1260 Infinity Quaternary LC System, equipped with an Agilent 6130 MS spectrometer. Samples were hydrolyzed in $6 \mathrm{~N} \mathrm{HCl}$ at $105^{\circ} \mathrm{C}$ for $24 \mathrm{~h}$ and were then diluted in $0.1 \mathrm{~N} \mathrm{HCl}$ for analysis. Amino acids including aspartic acid (D), serine $(S)$, glycine $(G)$, and arginine $(R)$ were detected and quantified by reacting with $o$-phthalaldehyde (OPA) derivatization solution in borate buffer. A reverse-phase Agilent C18-Extend column $(3.5 \mu \mathrm{m}$, $4.6 \times 75 \mathrm{~mm}$ ) was used at $40{ }^{\circ} \mathrm{C}$ column temperature. The flow rate of the elution gradient ( $0 \mathrm{~min}, 20 \%$ buffer B; $5.0 \mathrm{~min}, 60 \%$ buffer B; 9.5 min, $60 \%$ buffer B; 10.0 min, $20 \%$ buffer B; where buffer A: 10 $\mathrm{mM}$ sodium phosphate dibasic $+10 \mathrm{mM}$ sodium tetraborate, $\mathrm{pH} 8.2$ and buffer B: $45 \%$ methanol, $45 \%$ acetonitrile, $10 \%$ water by volume) was $1 \mathrm{~mL} / \mathrm{min}$. A standard curve was obtained by running different concentrations of G, R, G, D, and S solution ranging from 100 to $1000 \mu \mathrm{M}$. Dynamic light scattering (DLS) was performed using a Wyatt, DynaPro NanoStar at $1 \mathrm{mg} / \mathrm{mL}$. Fluorescence images were acquired on an Olympus FV1000 inverted confocal microscope with hyperspectral acquisition capabilities, using specific wavelength laser diodes as the excitation source and the manufacturer's recommended filter settings. For solution studies, a drop of a $20 \mathrm{mg} / \mathrm{mL}$ dye-loaded nanoparticle solution was deposited on a glass slide. Measurements in well plates were performed using a microplate reader (PowerWave HT, Bio-Tek).

Stability Measurements. The stability of particle sizes over time was evaluated by incubating aliquots of particles at $1 \mathrm{mg} / \mathrm{mL}$ in $37^{\circ} \mathrm{C}$ PBS. The concentration corresponded to the peptide content that resulted in optimum performance as determined through aggregation tests. The size stability study was conducted in PBS due to large amounts of interference from protein aggregates, regardless of the particle type and concentration tested. The samples were removed at $30 \mathrm{~min}$ and 1,2 , and $3 \mathrm{~h}$ time points and measured with DLS to 
Scheme 1. (a) Schematic Representation of Chemical Reactions Leading to PLGA- $b$-PEG-GRGDS Nanoparticles or DuallyFunctionalized PLGA- $b$-PEG-GRGDS/Biotin Micelles; (b) Coumarin 6-Labeled PLGA- $b$-PEG-GRGDS Nanoparticles in PBS at $20 \mathrm{mg} / \mathrm{mL}$
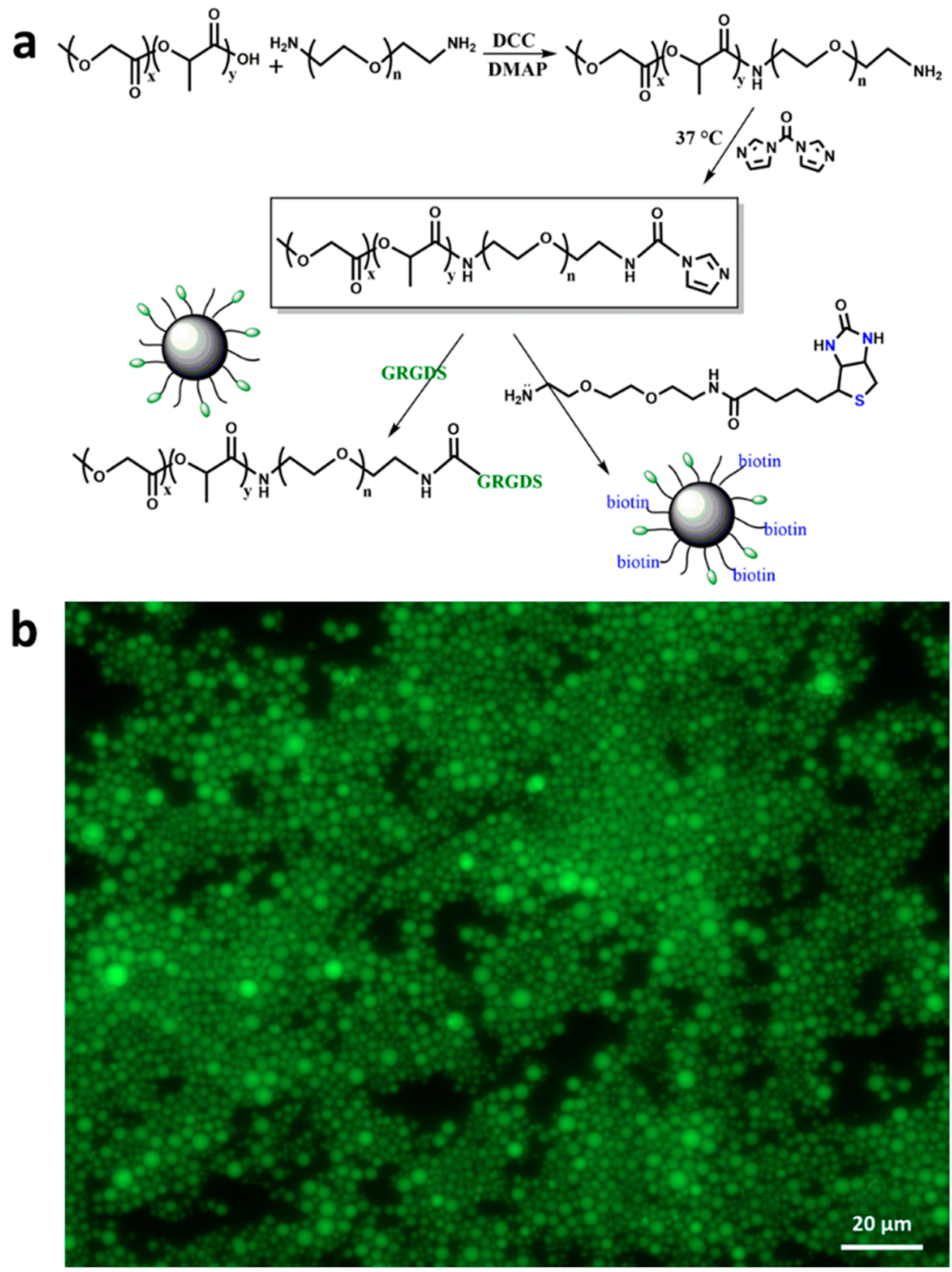

detect any changes in size over the incubation period. Hydrophobic dye release was evaluated by incubating $20 \mu \mathrm{L}$ aliquots of DiD-labeled nanoparticles at $10 \mathrm{mg} / \mathrm{mL}$ in PBS to $180 \mu \mathrm{L}$ of FBS, yielding $1 \mathrm{mg} /$ $\mathrm{mL}$ of DiD-labeled nanoparticles in FBS. These samples were incubated at $37^{\circ} \mathrm{C}$ and agitated at $200 \mathrm{rpm}$ on a shaker. The samples were removed at $1 \mathrm{~h}, 3 \mathrm{~h}, 5 \mathrm{~h}, 1$ day, and 5 days, and three samples were analyzed per time point. The samples were centrifuged at 30000 $\mathrm{rcf}$ at $4{ }^{\circ} \mathrm{C}$ for at least $3 \mathrm{~h}$ to separate particles from the serum, and the pellet was collected and lyophilized. When dry, the pellets were dissolved in $250 \mu \mathrm{L}$ of DMSO and sonicated for $1 \mathrm{~h}$ and, then, measured in a Tecan microplate reader at $625 \mathrm{~nm}$ excitation and 665 $\mathrm{nm}$ emission. A standard curve and $t=0$ were from $20 \mu \mathrm{L}$ of DiDlabeled nanoparticles at $10 \mathrm{mg} / \mathrm{mL}$ that were lyophilized and dissolved in DMSO. Blank samples of pure serum were also measured, and readings were found to be at least an order of magnitude below that of the least concentrated fluorescent nanoparticle standards made from the DiD-labeled stock solution.
The remaining stability studies for gold- and biotin-labeled particles were conducted in PBS, due to the nature of the measurements: the residual amount of serum was difficult to allow for Au-NPs quantification in THF and could skew absorbance readings; similarly, dissolution of protein in NMR could obscure peaks used to determine biotin loss. Stability of gold-labeled nanoparticles was evaluated using UV-vis spectroscopy (Varian Cary 50). Following incubation at 1 $\mathrm{mg} / \mathrm{mL}$ in $37{ }^{\circ} \mathrm{C}$ PBS overnight, gold-labeled nanoparticles were centrifuged to separate them from the supernatant. Water was removed from the supernatant using rotary evaporation, and THF was added to both the vial and the dried pellet at the same concentration. The THF solutions were then measured using UV-vis spectroscopy. Similarly, biotin-labeled particles were incubated at $2 \mathrm{mg} / \mathrm{mL}$ (double the concentration of the gold and DiD-labeled NPs to keep peptide content constant) in PBS at $37{ }^{\circ} \mathrm{C}$ for $3 \mathrm{~h}$ or overnight before centrifugation, after which the pellet and supernatant were lyophilized to remove water. The biotin remaining in the pellet was dissolved in 
deuterated DMSO and quantified using NMR spectroscopy by integration of characteristic peaks (biotin urea protons at 6.43 and $6.36 \mathrm{ppm}$, lactide protons at $5.20 \mathrm{ppm}$, and glycolide protons at 4.83 $\mathrm{ppm})$. As the nanoparticles were comprised of GRGDS-functionalized PEG-PLGA, biotin-labeled PEG-PLGA, and potentially, unreacted PEG-PLGA, significant loss of the biotin moiety only would lead to an increase in the polymer/biotin peak ratio.

Statistical Analysis. Data were analyzed using STATA (version 13.1) software. Numerical variables were expressed as medians with percentiles $(\mathrm{Q} 1-\mathrm{Q} 3)$. Categorical variables were summarized as frequencies and percentages. Comparisons among the three groups were performed using the Kruskal-Wallis test for the numerical variables and the chi-square test for the categorical ones. Additionally, a second analysis was performed, by combining the animals of the second and third group (all animals receiving nanoparticle-GRGDS treatment) into a single group and comparing it to the control group (saline), using the Mann-Whittney $U$ test and the Fischer's exact test for the numerical and the categorical variables, respectively. Finally, multivariable analysis was performed in order to identify potential predictors of outcomes, controlling for possible confounding variables. A $p$-value of less than 0.05 was defined as the level of statistical significance.

\section{RESULTS AND DISCUSSION}

Nanoparticle Synthesis, Characterization, and Stability. PLGA- $b$-PEG nanoparticles were prepared from inexpensive, commercially available materials and were functionalized with GRGDS moieties (Scheme 1). The synthesized nanoparticles were designed to contain a stable amide bond linking PLGA and PEG as well as an amine end-group that enables the formation of stable urea products when further functionalized (Scheme 1). Two batches of polymer, Batch 1 and Batch 2, were synthesized with the methods noted above, with Batch 2 used for additional size and stability characterization of labeled and unlabeled nanoparticles. Characterization by gel permeation (GPC) and nuclear magnetic resonance (NMR) confirmed the molar mass and weight ratio of the polymer (Figures S1 and S2). The molar masses of the polymer blocks were designed to be $\sim 10 \mathrm{kDa}$ for PLGA and $6 \mathrm{kDa}$ for PEG. A larger hydrophilic/hydrophobic ratio than previously reported ${ }^{2,31}$ was chosen to increase the exposure of the hydrophilic target group (GRGDS) while decreasing the rate of assembly of the hydrophobic PLGA cores. Amino acid analysis showed $13.3 \pm 2.7 \mu$ mol GRGDS/g of polymer in Batch 1 according to a standard curve (Figure S3a). Dynamic light scattering (DLS) of the unlabeled PLGA$b$-PEG-GRGDS nanoparticles showed a mean diameter of 575 $\pm 62 \mathrm{~nm}$ (Figure S4), consistent with the literature. ${ }^{31}$ The nanoparticles were also loaded with coumarin 6 and imaged under a fluorescence microscope. Spherical nanostructures with a diameter of $\sim 1-2 \mu \mathrm{m}$ were obtained, showing efficient encapsulation of the dye (Scheme 1). DLS measurements on coumarin 6-labeled nanoparticles resulted in a similar mean diameter of $1393 \pm 325 \mathrm{~nm}$ (Table S1). These coumarin 6labeled nanoparticles were not used in the subsequent in vitro and in vivo studies.

Significant changes in particle size were observed depending on the labeling method used. Additional size and stability characterization was completed on Batch 2 particles to evaluate the effect of biotin labeling, dye or gold nanoparticle encapsulation. The sizes, obtained via DLS at two different concentrations, are summarized in Table S1. Encapsulation of DiD had a negligible effect on mean particle diameter relative to its unlabeled counterpart $(334 \pm 2$ and $328 \pm 3 \mathrm{~nm}$ at 1 $\mathrm{mg} / \mathrm{ml}$, respectively), while biotin labeling increased the $z$ - average diameter to $429 \pm 6 \mathrm{~nm}$. The diameter of the gold nanoparticles $(374 \pm 9 \mathrm{~nm}$ at $1 \mathrm{mg} / \mathrm{mL})$ differed significantly according to which concentration they were measured, possibly due to the effect of multiple scattering, which is consistent with the observation of smaller sizes in the more concentrated sample. In the dilute sample, encapsulation of gold nanoparticles resulted in a $455 \pm 17 \mathrm{~nm} z$-average size, larger than the DiD-labeled or unlabeled particles and significantly more polydisperse and multimodal than all labeled particles (Figure S5).

A potential challenge faced in the field of injectable hemostats is the occurrence of complement activation-related pseudoallergy (CARPA), which results in significant changes in heart rate, blood pressure, and concentration in blood gases. ${ }^{35}$ While this complement response occurs in both rodents and larger animal models (such as dogs and pigs), it is significantly milder in small animals and frequently overlooked. ${ }^{36}$ Consequently, testing in higher order species is a necessary next step.

In addition to the size and polydispersity measurements of nanoparticles with different labels previously noted, measurements on particle surface charge were conducted. It was found that the zeta potential of the unlabeled particles was measured to be largely neutral $(-0.22 \pm 1.63 \mathrm{mV})$, unlike the highly cationic poly(lysine) block used in similar hemostatic nanoparticles. ${ }^{36}$ The biotin-labeled particles exhibited a similar trend $(-1.23 \pm 0.79 \mathrm{mV})$, while all other labeling methods involved encapsulation of a hydrophobic moiety and no further modifications to the hydrophilic corona.

The stability of labeled nanoparticles was evaluated through two methods: the size stability of the nanoparticles in PBS at $37{ }^{\circ} \mathrm{C}$ and the retention of the label. Overall, most of the particles remained approximately the same size over a period of $3 \mathrm{~h}$ (the duration of the in vivo experiment), with the largest change in size, an increase of around $15 \%$, observed in the gold-labeled nanoparticles (Figure S6). All other labeling methods resulted in an absolute size change of less than $5 \%$, similar to the unlabeled particles.

Label retention tests showed that the various labels were retained on or in the nanoparticles for a duration longer than that of the in vivo experiments. In $\mathrm{DiD}$ labeled particles, $4.4 \%$ of the hydrophobic dye was liberated from the particles over 5 $\mathrm{h}$ of incubation in serum (Figure S7a) and the majority over several days, consistent with the release of hydrophobic cargo shown in the literature. ${ }^{37}$ The UV-vis spectra of the supernatant from centrifuged Au-labeled NPs overnight showed no peaks, which were indicative of no leaching or an unmeasurably low concentration of naked gold nanoparticles. UV-vis measurements of the pellet approximately 1 week after synthesis likewise did not exhibit a sharp peak characteristic of $5 \mathrm{~nm}$ gold nanoparticles $(517 \mathrm{~nm})$ and instead showed a broader peak shifted to higher wavelengths, indicative of gold aggregates inside the micelle or entrapment of gold nanoparticles (Figure S8). As biotin was covalently attached to the polymer with a stable urea bond (identical to the one connecting GRGDS to PEG), it was not expected to degrade during the course of the experiment. NMR was used to confirm this at a $3 \mathrm{~h}$ time point and overnight, using the biotin/lactide and biotin/glycolide peak area ratio (Figure S9 and Table S2). As can be seen, this phenomenon is largely absent (with less than a $3 \%$ difference) in samples tested both at $3 \mathrm{~h}$ and overnight. 
a

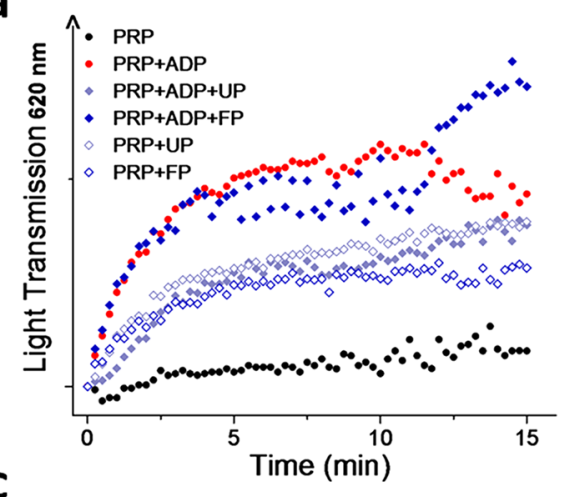

C

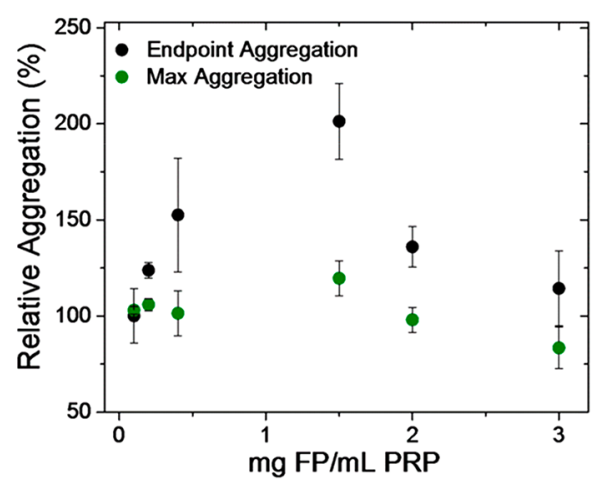

b

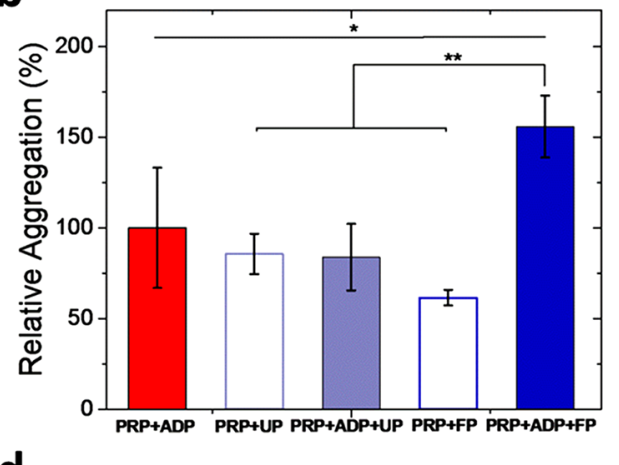

d

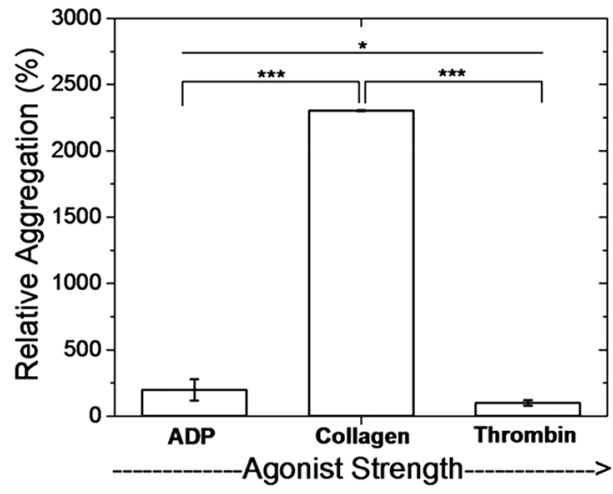

Figure 1. Particles modulate platelet aggregation in vitro. A microplate reader was used to monitor the rate of platelet aggregation in platelet rich plasma (PRP) at $620 \mathrm{~nm}$. The increase in transmission reflects the generation of platelet aggregates. (a) Aggregation curves from ADP activated PRP with and without nanoparticles. (b) Relative platelet aggregation was increased in samples activated with ADP and containing FP (PLGA-bPEG-GRGDS), compared to PRP + ADP or PRP + FP controls. (c) Different concentrations of FP were tested to identify a peak in platelet aggregation. Aggregation was maximized at $1.5 \mathrm{mg} / \mathrm{mL}$ according to end point and maximum aggregation. (d) Comparing platelet agonists, relative aggregation was most increased in collagen-activated PRP. Aggregation was normalized with respect to PRP + agonist (PC in the case of thrombin to prevent coagulation from occurring). ( $N=3$; ns: $p>0.05$; $: p \leq 0.05$; **: $p \leq 0.01$; ***: $p \leq 0.001$; statistical significance determined by oneway analysis of variance with Tukey post hoc comparisons).

In Vitro Coagulation and Platelet Aggregation. Increased platelet aggregation and reduced coagulation rates were observed during microplate monitoring of platelet aggregation (Figures 1, S10, and S11) and coagulation (Figure S12) with functionalized particles. Sodium citrate anticoagulated human platelet rich plasma (PRP), whole blood devoid of red blood cells (RBCs), was used for all measurements. Data was transformed from absorbance to transmission, resulting in an increase in transmission during platelet aggregation and a decrease in transmission during coagulation. ${ }^{38}$ Coagulation occurred in all calcium chloride activated systems but was reduced in those systems containing unfunctionalized particles (UP: PLGA- $b$-PEG) and functionalized particles (FP: PLGA- $b$-PEG-GRGDS) relative to the control (Figure S12). The reduced extent of coagulation has been observed in vitro in systems containing PLGA nanoparticles. $^{39}$ Additionally, RGD has been associated with increased calcium levels in some cell systems ${ }^{40}$ and is thought to have some affinity for calcium via aspartic acid ${ }^{41}$ which could impact calcium levels available for coagulation. In the nanoparticles (labeled and unlabeled) synthesized from Batch 2 , coagulation rate was not impaired, and neither was relative coagulation (\%) (Figure S13). Analysis was continued for platelet aggregation.

Functionalized particles showed an enhancement in platelet aggregation compared to controls (PRP + ADP) and systems containing unfunctionalized particles (Figure 1a). Adenosine diphosphate (ADP) served as an agonist for platelet activation and subsequent aggregation. Agitation of the well plate was performed by the plate reader between readings to prevent platelet aggregates settling from impacting the transmission measurements. Aggregation was 1.6 times higher when FP were added to ADP-activated PRP $([\mathrm{ADP}]=10 \mu \mathrm{M})$, while a decrease in aggregation was observed when UP were added (0.8 times lower than PRP $+\mathrm{ADP}$ ) relative to controls (Figure 1b). Further study of FP concentrations showed minimal impact on aggregation at low concentrations and a deleterious effect on aggregation at high concentrations, with the highest aggregation values occurring at $1.5 \mathrm{mg} \mathrm{FP} / \mathrm{mL} \mathrm{PRP}$, regardless of the metric used to quantify aggregation (maximum aggregation or end point aggregation values) (Figures 1c). Platelet aggregation tests were also performed with nanoparticles synthesized from Batch 2 (labeled and unlabeled) (Figures S14 and S15). The results were consistent with those reported above, acknowledging the differing GRGDS conjugation efficiencies between Batch 2 and Batch 1 (16.3 vs 13.3 $\mu \mathrm{mol}$ GRGDS/g, Figure S3b), resulting in enhanced platelet aggregation. Overall, DiD-labeled and unlabeled particles resulted in a larger increase in aggregation for the same concentration of particles delivered, possibly due to the different size and surface area/mass ratio of the particles relative to their gold- and biotin-labeled counterparts.

Aggregation activated by other agonists (collagen and thrombin) highlighted agonist-dependent aggregation that 

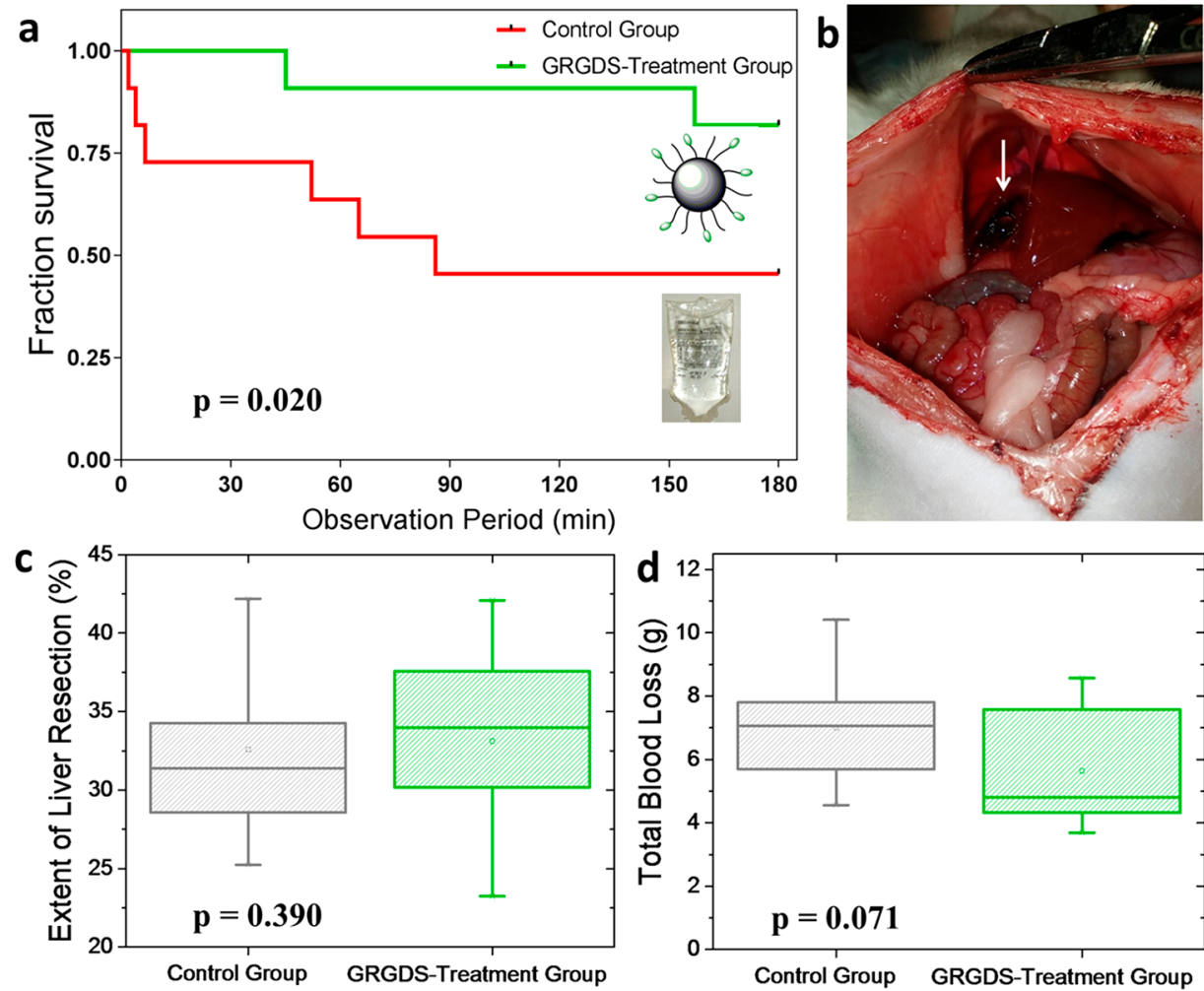

Figure 2. (a) Kaplan-Meier curves of PLGA-b-PEG-GRGDS nanoparticles (green) and saline (red) treatments. (b) Clots formed in the liver (white arrow). (c) Percent of median liver resected. (d) Total blood loss of the two treatments. The delivery of nanoparticles significantly prolonged the survival $(p=0.020)$ as well as the survival time $(p=0.020)$ for similar liver resection percentages $(\sim 33 \%)$.

would require further molecular investigations to generate a complete mechanism of action (Figures $1 \mathrm{~d}$ and S10). In brief, collagen-activated PRP showed significantly higher aggregation (23 times higher) when FP were used, compared to UPcontaining systems and controls, while the FP have an insignificant effect on platelet concentrate (PC) activated by thrombin (Figures 1d and S5). Knowledge of the window of functional activity is necessary when this treatment strategy is considered for larger animals or for clinical trials in humans. On the basis of these in vitro results and estimated blood volumes for rats weighing 300-400 g, ${ }^{42}$ a dosage of $\sim 1.5 \mathrm{mg}$ of GRGDS-particles/mL blood was used for the subsequent in vivo studies.

Therapeutic Performance of GRGDS Nanoparticles. Systemic delivery of the GRGDS-functionalized hemostatic particles to rodents, injured by a lethal liver resection, resulted in clotting augmentation, with reduced blood loss and a significant increase in survival rate, compared to the salinetreated animals (Figure 2). Specifically, intravenous injection of PLGA- $b$-PEG-GRGDS nanoparticles immediately after the liver resection resulted in $82 \%$ rodent survival during the $3 \mathrm{~h}$ experimental time window compared to the saline group ( $45 \%$ survival) (Figure 2a,b) for similar liver resection percentages $(\sim 33 \%)$ (Figure $2 \mathrm{c})$. This increase in survival rates is among the highest reported in the literature for lethal injuries in rats $^{27,31}$ and is being recorded for the longest observation time ( $3 \mathrm{~h}$ vs $1 \mathrm{~h}$ ). Considering saline treated rats, 5 out of 11 animals survived after liver resection while 9 out of 11 GRGDS-nanoparticle treated rats survived through the same observation time. Only two of the animals that received the hemostatic nanoparticles died at 45 and $157 \mathrm{~min}$, respectively (Figure 2a), suggesting that in the majority of rodents hemostasis was achieved faster in the presence of PLGA- $b$ PEG-GRGDS compared to saline-treated animals (Table 1). Additionally, the median blood loss volume decreased from 7.1 to $4.8 \mathrm{~g}$, a $2.3 \mathrm{~g}$ decrease in blood loss with the nanoparticle treatment (Figure 2d). Results showed that nanoparticle delivery significantly prolonged survival $(p=0.020)$ as well as the survival time $(p=0.015)$ following a lethal injury (Table 2 ), compared to delivery of an equal volume of saline.

Table 1. Univariate Statistical Analysis Comparing the 3 Different Groups Using the Kruskal-Wallis Non-Parametric Test

\begin{tabular}{|c|c|c|c|c|}
\hline variable & saline treated & GRGDS only & $\begin{array}{l}\text { GRGDS with } \\
\mathrm{F} / \mathrm{B} / \mathrm{G}^{a}\end{array}$ & $\begin{array}{c}p \text { - } \\
\text { values }\end{array}$ \\
\hline $\begin{array}{l}\text { number of } \\
\text { animals }\end{array}$ & 11 & 11 & $9^{b}$ & $\mathrm{n} / \mathrm{a}$ \\
\hline mortality & $6(55 \%)$ & $2(18 \%)$ & $0(0 \%)$ & 0.020 \\
\hline $\begin{array}{l}\text { survival time } \\
\text { (min) }\end{array}$ & $86(6.5-180)$ & $\begin{array}{l}180 \\
(180-180)\end{array}$ & $\begin{array}{l}180 \\
(180-180)\end{array}$ & 0.015 \\
\hline blood loss (g) & $7.1(5.7-7.8)$ & $4.8(4.3-7.6)$ & $\begin{array}{l}7.3^{a} \\
(5.7-8.5)\end{array}$ & 0.071 \\
\hline $\begin{array}{l}\text { total liver weight } \\
(\mathrm{g})\end{array}$ & $\begin{array}{l}11.6 \\
(9.4-13.1)\end{array}$ & $\begin{array}{l}10.1 \\
(8.8-12.7)\end{array}$ & $\begin{array}{l}13.9 \\
(12.8-17.4)\end{array}$ & 0.028 \\
\hline $\begin{array}{l}\text { resected liver } \\
\text { weight }(\mathrm{g})\end{array}$ & $3.7(3.0-4.3)$ & $3.2(3.1-3.9)$ & $5.2(4.4-5.7)$ & 0.014 \\
\hline $\begin{array}{c}\text { median blood } \\
\text { loss/median } \\
\text { liver weight }\end{array}$ & 0.61 & 0.48 & 0.53 & $\mathrm{n} / \mathrm{a}$ \\
\hline $\begin{array}{l}\text { liver resected } \\
(\%)\end{array}$ & $\begin{array}{l}31.5 \\
\quad(28.6-34.3)\end{array}$ & $\begin{array}{l}34.0 \\
\quad(30.2-37.5)\end{array}$ & $\begin{array}{l}35.2 \\
\quad(32.9-38.9)\end{array}$ & 0.390 \\
\hline male gender & $5(45 \%)$ & $4(36 \%)$ & $9(100 \%)$ & 0.007 \\
\hline
\end{tabular}

${ }^{a} \mathrm{~F} / \mathrm{B} / \mathrm{G}$ : fluorescent dye, biotin, gold nanoparticles. ${ }^{b}$ All male animals. 
Tracer addition to GRGDS-nanoparticles did not impact their performance in vivo, with all three groups, fluorescent dye, biotin, and gold labeling, achieving 100\% (9/9) survival and 180 min survival time. No animal that received the tracercontaining nanoparticle treatment succumbed to their injuries during the experimental time frame. This suggests that the interactions of the nanoparticles with blood components are not significantly affected by the introduction of hydrophobic markers (DiD fluorescent dye or Au-NPs) or a hydrophilic marker (biotin) for the tested animal model during the $3 \mathrm{~h}$ observation window. However, animals that were treated with the nanoparticles including a tracer did have the greatest blood loss (Table 2). Though in vitro coagulation tests with different labeling methods did not show an appreciable decrease in coagulating ability (Figure S13), platelet aggregating capability appeared to be affected and could lead to some of the observed differences. Additionally, all the tracer-containing functionalized particle treated animals (GRGDS with $\mathrm{F} / \mathrm{B} / \mathrm{G}$ ) were male compared to a mixed sex cohort in the control groups. While gender was not necessarily a metric of comparison between groups, the difference in weight (and therefore larger animal blood volume) between male and female rodents could convolute the use of blood loss as an independent metric for nanoparticle performance and thus was listed alongside results such as blood loss and survival time. Similarly, liver weight was also larger with groups that contained more male animals. Since liver resection percentages were kept at approximately $33 \%$, total liver weight indicated how much liver weight had been removed. In fact, when blood loss was normalized to the median total liver weight for each test group, both GRGDS and tracer-containing GRGDS nanoparticle groups had less relative blood loss. Median blood loss per median total liver weight was 0.61 for the saline-treated group, 0.48 for the GRGDS particletreated group, and 0.53 for the tracer-containing GRGDS particle-treated group.

All groups (saline control, GRGDS only, GRGDS with dyes) were analyzed independently in the first round of univariate analysis (Table 1). Univariate analysis using the MannWhitney $\mathrm{U}$ test was performed using the combined GRGDSnanoparticle groups (Table 2 ). The mortality was significantly

Table 2. Univariate Analysis Comparing the Control Group with the Combined GRGDS Group Using the MannWhitney U Test

\begin{tabular}{llll}
\multicolumn{1}{c}{ variable } & \multicolumn{1}{c}{ saline treated } & \multicolumn{1}{c}{ all GRGDS } & $\begin{array}{c}p \text { - } \\
\text { values }\end{array}$ \\
number of animals & 11 & 20 & $\mathrm{n} / \mathrm{a}$ \\
mortality & $6(55 \%)$ & $2(10 \%)$ & 0.012 \\
survival time (min) & $86(6.5-180)$ & $180(180-180)$ & 0.005 \\
blood loss (g) & $7.1(5.7-7.8)$ & $5.9(4.4-7.8)$ & 0.389 \\
total liver weight (g) & $11.6(9.4-13.1)$ & $12.6(9.8-14.9)$ & 0.457 \\
resected liver weight $(\mathrm{g})$ & $3.7(3.0-4.3)$ & $3.9(3.2-5.6)$ & 0.265 \\
liver resected (\%) & $31.5(28.6-34.3)$ & $34.7(30.6-38.0)$ & 0.322 \\
male gender & $5(45 \%)$ & $13(65 \%)$ & 0.450 \\
\hline
\end{tabular}

( $p=0.012)$ reduced compared to the saline-treated group while the survival time was likewise increased when treated with GRGDS-functionalized nanoparticles $(p=0.005)$. Multivariable analysis was also performed to establish survival predictors for the treatment. Analysis showed that the nanoparticle treatment is an independent predictor for survival (Table S3). Parameters related to body weight, such as sex or liver weight, were not significant predictors for mortality or survival time, though liver weight was a significant predictor in the resulting blood loss.

Near-Infrared Imaging in Small Animals. DiD (a nearinfrared dye)-labeled nanoparticles were visible in organs of injured rodents at wavelengths within the near-infrared window of biological tissues. Dyes with excitation and emission wavelengths above $700 \mathrm{~nm}$ allow for increased tissue penetration depth and reduced background signal from biological molecules. ${ }^{20}$ NIR-based fluorescence imaging has been beneficial in small animal studies for localization and distribution of molecules or particles in real time in live animals. It has also been used in humans for intradermal or subcutaneous imaging. In this study, $\mathrm{DiD}$ was present in the core of GRGDS-containing nanoparticles and visualized with confocal imaging of tissue sections. Whole organ quantification of $\mathrm{DiD}$ concentrations was used to measure biodistribution of particles throughout the body. A high percentage of the injected nanoparticles containing $\mathrm{DiD}$ was found in the clots present at the liver injury site, implying that the injected materials are capable of integrating into the clot. Confocal imaging of tissue sections from different organs allowed for preliminary tracking of the nanoparticles by collecting the emission spectra using the hyperspectral channel of a confocal microscope, giving an initial estimate of biodistribution. A PBS suspension of DiD-labeled PLGA- $b$-PEG-GRGDS nanoparticles showed emission at $665 \mathrm{~nm}$ (Figure S16). The resected livers from each animal (Figure S17), as well as tissue sections from different organs of a saline-treated animal, were used as negative controls confirming the absence of any peak in the NIR region. Imaging of liver-excised clot sections verified the existence of DiD-labeled nanoparticles inside the clots (Figures $3 a-c$ and S18) without interference from tissue autofluorescence (Figure 3d-f). A small amount of DiD-labeled nanoparticles was found in the heart and kidneys (Figures S19 and S20), while a higher number of particles were found in spleen, lungs, and remnant liver (Figures S21-S23). To quantify this, chemical analysis of whole organs was performed in order to obtain biodistribution values for the entire organ, since there are major weight differences among different organs.

Results confirmed a minimal number of nanoparticles (as a percent of all particles injected) in the heart $(0.6 \pm 0.2 \%)$ and kidneys $(1.1 \pm 0.2 \%)$ and a relatively smaller number in the spleen $(11 \pm 2 \%)$ (Figure S24) compared to the liver and clot tissue. The spleen is the organ that, among its other roles, stores a significant percentage of platelets, possibly accounting for the nanoparticles seen inside this organ. $19 \pm 2 \%$ of the nanoparticles were found in the lungs. Relatively high fractions of nanoparticles in the spleen and lungs can be attributed to their large size, which can be easily entrapped in the lungs and spleen. ${ }^{43}$ It is important to stress the fact that none of the animals experienced respiratory complications during the observation time. However, further experiments need to be done to evaluate more physiologic responses and verify the absence of microemboli or respiratory distress, which could occur as a result of the micron-sized particles occluding smaller vasculature like capillaries or arterioles (diameters 5-12 $\mu \mathrm{m}) .{ }^{44}$ Clots were found to contain $30 \pm 2 \%$ of the particles, supporting the hypothesis that the nanoparticles accumulate and act at the injury site, possibly promoting clot formation (Figure 3). Blood was not included among the compartments selected to quantify particle concentration. Additionally, $38 \pm$ 

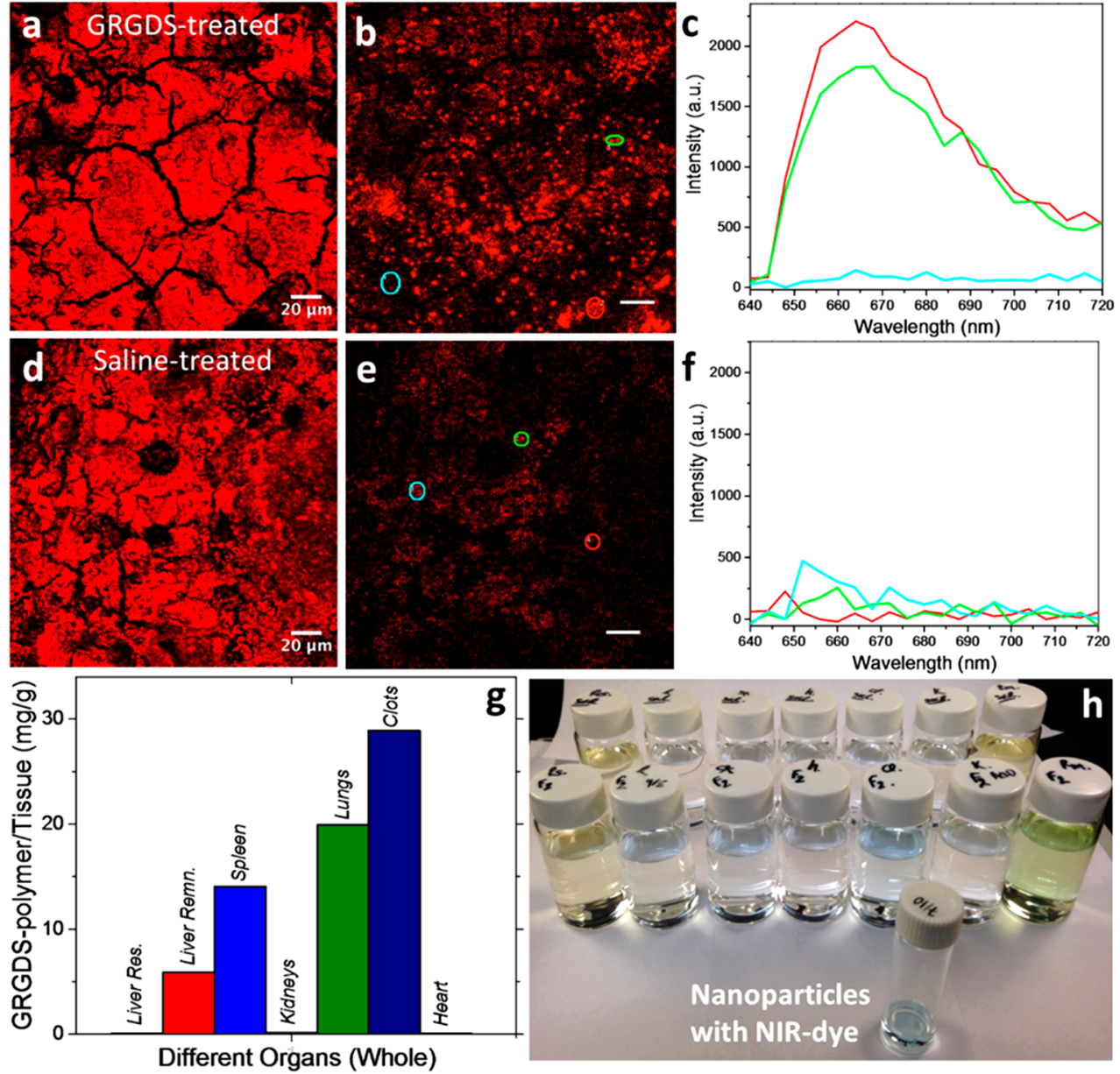

Figure 3. Imaging of liver-excised clot sections of animals treated with saline (negative control) and DiD-labeled nanoparticles. (a) Overall emission of PLGA(DiD)- $b$-PEG-GRGDS treated rats collected in the range of $640-720 \mathrm{~nm}$. (b) A single image from the lambda scan, corresponding to the 660-665 nm spectral region where $\mathrm{DiD}$ displays its maximum emission, showing the presence of nanoparticles inside the liver clots. (c) Emission spectra extracted from the hyperspectral channel. The colors match the circled regions shown in (b). (d) Overall emission of saline-treated rats collected in the range of $640-720 \mathrm{~nm}$. (e) A single image from the lambda scan, corresponding to the 660-665 nm spectral region. (f) Emission spectra extracted from the hyperspectral channel, showing the absence of peaks in that area. The colors match the circled regions shown in (e). (g) Bar graph of GRGDS-polymer/g of tissue $(\mathrm{mg} / \mathrm{g})$ and $(\mathrm{h})$ images of acetonitrile solutions containing DiD dye extracted from whole organs containing intravenously injected PLGA(DiD)-b-PEG-GRGDS nanoparticles, showing high accumulation of the particles in the clots as well as in lungs, spleen, and the remnant liver. The yellow color of the liver extract denotes the presence of endogenous coumarins while the cyan color (positive control in the front) is due to the DiD-loaded nanoparticles. Heart and kidneys are transparent due to the absence of nanoparticles, while the remnant liver is green due to the combination of yellow and cyan. From left to right: Resected liver, lungs, spleen, heart, clots, kidneys, and remnant liver. The vials in the back row represent acetonitrile tissue extracts of a saline-treated rat, showing transparency in all the organs except the liver.

$2 \%$ of the nanoparticles were found in the remnant liver. This is expected, since clots were formed in the liver, and even though they were removed from the resected edges, undoubtedly some clots were left intact in the hepatic vasculature. Additionally, small blood vessels in the liver could retain some particles, though all the examined organs were washed multiple times with PBS to remove any remaining blood that could impact the result. The liver is also the largest (by volume and mass) of the examined organs and acts as the principal site of drug metabolism, detoxification, and filtration, potentially providing an explanation for the relatively high percentage of nanoparticles seen in the remnant liver. Controls in uninjured animals would be required to confirm this hypothesis.

Clots contained the highest concentration of nanoparticles, when normalized to the mass of the respective organ. To account for weight differences in the examined (dry) organs (spleen < lungs < clot $\leq$ heart < kidneys < resected liver < remnant liver), the fluorescence signal of each organ was converted to a polymer concentration, on the basis of a $\mathrm{DiD}$ calibration curve (Figure S25), resulting in values of GRGDSpolymer $(\mathrm{mg}) /$ tissue $(\mathrm{g})$ (Figure $3 \mathrm{~g}$ ). Results showed relatively small amounts of polymer in the resected liver and heart $(<0.05 \mathrm{mg} / \mathrm{g})$, while the kidneys contained $0.15 \mathrm{mg}$ of GRGDS-polymer/g of tissue. Remnant liver had $5.91 \mathrm{mg}$ of GRGDS-polymer/g of tissue, while $14.05 \mathrm{mg}$ of GRGDSpolymer/g of tissue was found in the spleen. The lungs contained $19.91 \mathrm{mg}$ of GRGDS-polymer/g of tissue. The highest amount of functionalized polymer per tissue was found in clots, with $28.87 \mathrm{mg}$ of GRGDS-polymer/g of tissue, confirming high accumulation at the injury site (Figure 3g,h).

Biotin Functionalization for Immunohistochemistry Labeling. GRGDS nanoparticles were shown to be amenable to immunohistochemistry (IHC) by the addition of biotin 

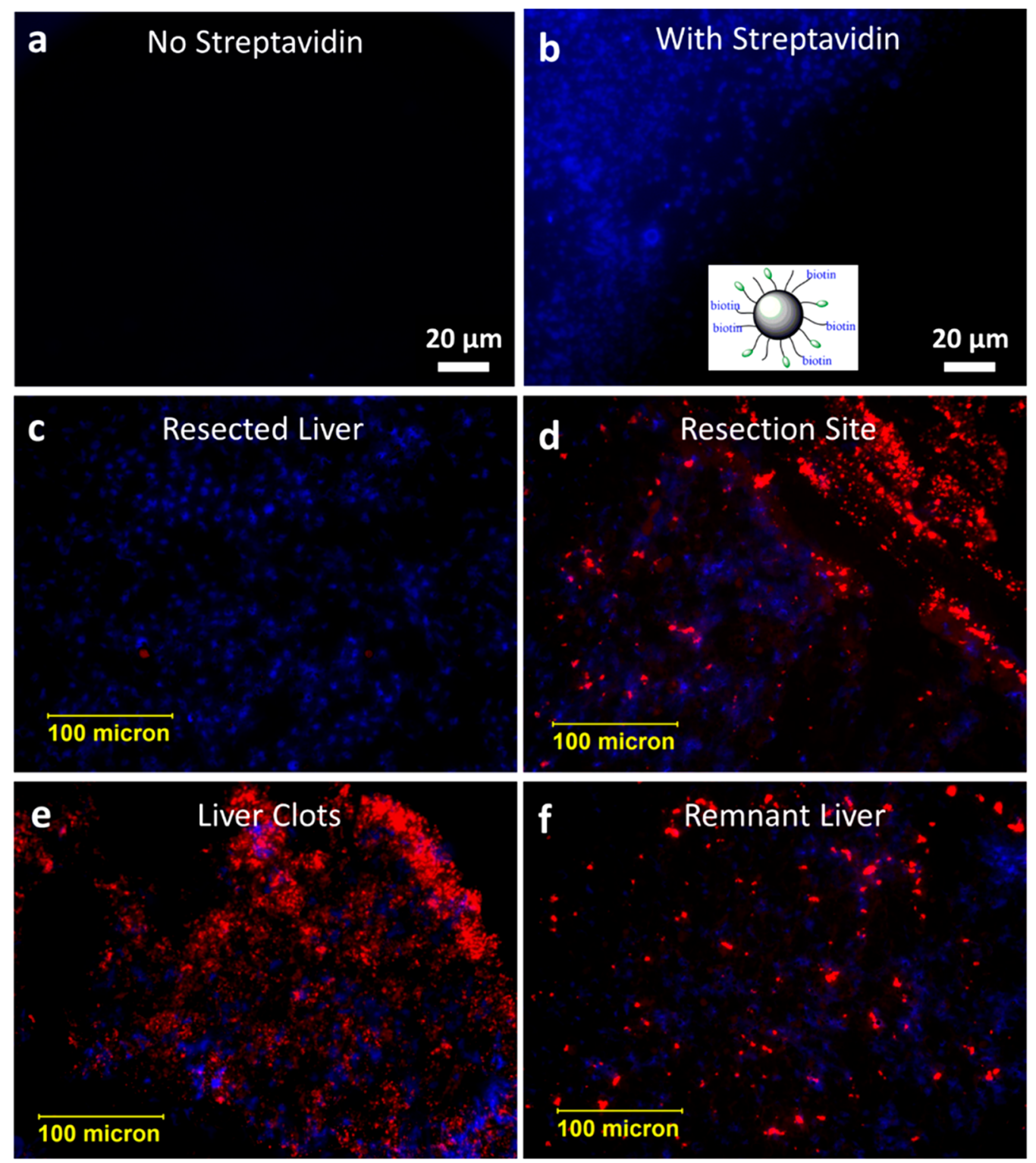

Figure 4. PLGA-b-PEG-GRGDS/biotin nanoparticles (a) in the absence and (b) in the presence of Alexa 350-conjugated streptavidin (blue). Imaging was performed at $442 \mathrm{~nm}$ after three rounds of centrifugation in order to remove unconjugated streptavidin. (c) Resected liver before systemic nanoparticle delivery. Accumulation of GRGDS/biotinylated nanoparticles (d) near the resection site between injured liver/clots, (e) in blood clots, or (f) in remnant liver, following systemic injection. The blue color represents DAPI staining of DNA in immune and other bloodborne cells while the red color denotes the biotin/streptavidin-HRP complexes which were amplified using tyramide fluorescence signal amplification of $\mathrm{Cy} 3$.

functionality into the PEG corona and subsequent labeling with streptavidin conjugated dyes following histology sectioning procedures. IHC serves as an analysis method that can identify interactions between antigens in the tissue and the nanoparticles after following biodistribution and integration into blood clots or other tissues. Labeling of nanoparticles with biotin from organs such as the liver, with sufficient staining, can localize particle accumulation within different parts of the liver architecture, improving an understanding of a mechanism of action or areas of aggregation or accumulation to a suborgan resolution. Biotin functionalization, known to be an immunologically inert labeling molecule, ${ }^{45}$ of the copolymer was confirmed by ${ }^{1} \mathrm{H}$ NMR (Figure S9). The two urea protons and the two methine protons (signature peaks) from the cyclic biotin structure were shown at 6.43 and $6.36 \mathrm{ppm}$ and at 4.31 and $4.20 \mathrm{ppm}$, respectively, while the amide bond is shown at
$7.85 \mathrm{ppm}^{46}$ The other peaks of the alkyl chain of biotin are hidden within the PEG and the PLGA signals. In vitro experiments using PLGA- $b$-PEG-GRGDS/biotin nanoparticles confirmed the presence of biotinylated particles by the addition of fluorescently labeled streptavidin (Figure 4). Fluorescently labeled streptavidin (Alexa 350 conjugate) was added to the nanoparticles, and imaging was performed after three rounds of centrifugation in order to remove unconjugated streptavidin. Fluorescence images of nanoparticles/ Alexa 350-streptavidin conjugates collected at $442 \mathrm{~nm}$ verified the conjugation of fluorescent streptavidin to biotinylated nanoparticles, in comparison with the neat nanoparticles (Figure 4a,b).

In addition, in vivo experiments using PLGA- $b$-PEGGRGDS/biotin nanoparticles verified the presence of biotinylated particles at the injury site. Biotin-containing nano- 
particles were detected using immunofluorescence microscopy in tissue sections obtained from the liver clot, remnant liver, and resected liver and were compared to tissue sections harvested from saline treated animals. Clots and the remnant liver of saline-treated animals, as well as the resected liver tissue of the GRGDS/biotin-treated animals, showed no biotin detection (Figure 4c). In contrast, substantial accumulation of nanoparticles was found in the remnant liver adjacent to the resection site (Figure 4d), while enhanced fluorescence signal was observed in the harvested clot tissues (Figure $4 \mathrm{e}$ ), following systemic injection of PLGA- $b$-PEG-GRGDS/biotin. Nanoparticles were also found in the remnant liver (Figure 4f), supporting the results from the fluorescently labeled nanoparticle experiment (Figure 3). Additional images of all the biotinylated/GRGDS particle-treated animals are included in Figures S26 and S27.

CT Imaging by Au Labeling. Similarly, PLGA- $b$-PEGGRGDS nanoparticles loaded with small-sized Au-NPs ${ }^{47,48}$ showed the capability to control bleeding (Tables 1 and 2), as well as the potential ability to be used as a contrast agent for CT scanning when incorporated into the injectable polymeric formulation. Naked gold nanoparticles have been shown to be ingested by human cells without causing acute cytotoxicity, $^{49-51}$ allowing for their unique X-ray pattern ${ }^{52}$ to be used for imaging purposes. GRGDS-nanoparticles containing hydrophobic gold ${ }^{53}$ were shown to be detected at the injury site using a CT scanner (Figure S8). Entrapment of Au-NPs into the hydrophobic PLGA cores does not permit any interactions with blood proteins, serving as a potential tool for whole body imaging, using a CT scan, ${ }^{54,55}$ and allowing for biodistribution studies in large animals. The surface plasmon resonance band of dodecanethiol-functionalized Au-NPs at $~ 517 \mathrm{~nm}$ (in THF) confirmed the formation of $\mathrm{Au}(0)$ (Figure S8a). Hydrophobic gold showed minimal aggregation upon its incorporation into the PLGA- $b$-PEG-GRGDS nanoparticles, with a slight shift of the surface plasmon resonance band at $\sim 528 \mathrm{~nm}$, after dissolution in THF (Figure S28). Shifts in the UV-vis spectrum are known to occur when gold nanoparticles are incorporated on different substrates including graphene, carbon nanotubes, or polymers. ${ }^{56,57}$ Quantitative analysis by a CT scan showed a negligible change in attenuation in the lungs upon injection with gold-loaded nanoparticles (compared to saline) but a noteworthy change in attenuation in clots, spleen, heart, and kidneys (Figure S8b). Remnant liver tissue was found to have a $25.7 \%$ increase in Hounsfield units (HU) compared to saline-treated animals, while the heart, clot, and kidney tissues had an increased attenuation of $45.8 \%$, $47.8 \%$, and $58.0 \%$, respectively. The spleen was found to have the highest change in attenuation, with a $243 \%$ increase in HU. A similar observation regarding the amount of Au-NPs in the spleen has been reported. ${ }^{58}$ While UV-vis measurements to characterize the stability of gold-labeled nanoparticles did not indicate significant leaching of naked gold, it is possible that some disassembly and liberation of gold occurred at a concentration too low to be detectable, and this could contribute to the discrepancies between the labeling methods. Moreover, the mean diameter and polydispersity of Au-labeled nanoparticles was significantly larger than their $\mathrm{DiD}$ or unlabeled counterparts, which could cause differences in the biodistribution of the particles, for example, high splenic accumulation, $^{43}$ an effect that may also have been observed in coumarin 6-labeled particles had they been selected for use in this study. Size stability studies also indicated that the gold- labeled nanoparticles could be less stable than the other labeling methods over the duration of the experiment (Figure S6), which could contribute to the observed disparity in biodistribution.

\section{CONCLUSIONS}

By using three different contrast dyes, GRGDS-functionalized PLGA- $b$-PEG nanoparticles were shown to function as a theranostic formulation, providing diagnostic and therapeutic activity. As a therapy, the nanoparticles accumulated in the clots formed in injured livers, which led to reduced blood loss and significantly improved the survival of injured rodents. As a diagnostic tool, imaging by NIR confocal microscopy, immunohistochemistry, and CT imaging were achieved by addition of $\mathrm{DiD}$, biotin functionality, and $\mathrm{Au}$-nanoparticles to the core or corona of the nanoparticles. While NIR imaging showed the highest particle accumulation in clots, followed by lungs and spleen, CT imaging showed a slightly different biodistribution map, with high particle accumulation in clots, kidneys (which had negligible signal in the fluorescence studies), and spleen but minimal accumulation in the lungs. NIR biodistribution results (apart from clots) are consistent with reported in vivo distribution of particles with sizes $>150$ $\mathrm{nm},{ }^{43}$ while the distribution of Au-labeled NPs likely reflects the additional instability and polydispersity that was not apparent in the other particles. Further controls and trials, including unfunctionalized particles in injured and uninjured rodents, would need to be performed to draw more conclusions regarding particle targeting and injury site-specific biodistribution claims. In vivo imaging of live animals would likewise need to be conducted to obtain a more concrete picture of the biodistribution over time with various labeling methods and to better understand the in vivo stability of nanoparticles. Finally, additional screening for complement cleavage fragments following nanoparticle incubation in blood or serum would offer more information on the possibility of CARPA occurrence prior to animal studies. Regardless, the continued therapeutic performance of the PLGA-PEG nanoparticles upon addition of diagnostic tracers is evidence of their potential as a theranostic system to manage and identify sources of internal bleeding. This study sets the stage for the development of hemostatic polymeric nanoparticles with additional diagnostic potential and further in vivo studies to determine mechanisms of action and whole organ biodistribution.

\section{ASSOCIATED CONTENT}

\section{S Supporting Information}

The Supporting Information is available free of charge on the ACS Publications website at DOI: 10.1021/acsbiomaterials.9b00054.

GPC, NMR, additional coagulation and aggregation curves, stability and release studies, and confocal and fluorescence images of nanoparticles in sectioned rodent organs (PDF)

\section{AUTHOR INFORMATION}

\section{Corresponding Authors}

*E-mail: bdolsen@mit.edu (B.D.O.).

*E-mail: GVELMAHOS@mgh.harvard.edu (G.V.).

ORCID $\odot$

Bradley D. Olsen: 0000-0002-7272-7140 


\section{Notes}

The authors declare no competing financial interest.

\section{ACKNOWLEDGMENTS}

This material is based upon work supported in part by the U.S. Army Research Office through the Institute for Soldier Nanotechnologies at MIT, under Cooperative Agreement Number W911NF-18-2-0048. R.K.A. was supported by the NIH Interdepartmental Biotechnology Training Program (NIH/NIGMS5T32GM008334). This research was supported by the U.S. Army Research Office under contract W911NF-13D-0001. The authors express their appreciation to the MIT Department of Chemistry Instrumentation Facility for the NMR instrumentation.

\section{ABBREVIATIONS}

PRP, platelet rich plasma; MRI, magnetic resonance imaging; CT, computed tomography; PLGA, poly(lactic-co-glycolic acid); PEG, poly(ethylene glycol); DMSO, dimethyl sulfoxide; HU, Hounsfield units

\section{REFERENCES}

(1) Vaezy, S.; Andrew, M.; Kaczkowski, P.; Crum, L. Image-Guided Acoustic Therapy. Annu. Rev. Biomed. Eng. 2001, 3 (1), 375-390.

(2) Forrest, J. H.; Finlayson, N. D. C.; Shearman, D. J. C. Endoscopy in Gastrointestinal Bleeding. Lancet 1974, 304 (7877), 394-397.

(3) Champion, H. R.; Bellamy, R. F.; Roberts, C. P.; Leppaniemi, A. A profile of combat injury. J. Trauma: Inj., Infect., Crit. Care 2003, 54 (5 Suppl), S13-S19.

(4) Lopera, J. E. Embolization in Trauma: Principles and Techniques. Seminars in Interventional Radiology 2010, 27 (1), 14-28.

(5) Behrens, A. M.; Sikorski, M. J.; Kofinas, P. Hemostatic strategies for traumatic and surgical bleeding. J. Biomed. Mater. Res., Part A 2014, 102 (11), 4182-4194.

(6) Carr, M. E., Jr. Monitoring of hemostasis in combat trauma patients. Mil. Med. 2004, 169 (12 Suppl), 11.

(7) Diegeler, A.; Thiele, H.; Falk, V.; Hambrecht, R.; Spyrantis, N.; Sick, P.; Diederich, K. W.; Mohr, F. W.; Schuler, G. Comparison of Stenting with Minimally Invasive Bypass Surgery for Stenosis of the Left Anterior Descending Coronary Artery. N. Engl. J. Med. 2002, 347 (8), 561-566.

(8) Wacker, F.; Boese-Landgraf, J.; Wagner, A.; Albrecht, D.; Wolf, K.-J.; Fobbe, F. Minimally invasive catheter implantation for regional chemotherapy of the liver: A new percutaneous transsubclavian approach. CVIR 1997, 20 (2), 128-132.

(9) Biere, S. S.; Cuesta, M. A.; van der Peet, D. L. Minimally invasive versus open esophagectomy for cancer: a systematic review and metaanalysis. Minerva Chir 2009, 64 (2), 121-133.

(10) de la Torre, R. A.; Bachman, S. L.; Wheeler, A. A.; Bartow, K. N.; Scott, J. S. Hemostasis and hemostatic agents in minimally invasive surgery. Surgery 2007, 142 (4), S39-S45.

(11) Xie, J.; Lee, S.; Chen, X. Nanoparticle-based theranostic agents. Adv. Drug Delivery Rev. 2010, 62 (11), 1064-1079.

(12) Kidwell, C. S.; Chalela, J. A.; Saver, J. L.; et al. Comparison of mri and ct for detection of acute intracerebral hemorrhage. JAMA 2004, 292 (15), 1823-1830.

(13) Nienaber, C. A.; von Kodolitsch, Y.; Petersen, B.; Loose, R.; Helmchen, U.; Haverich, A.; Spielmann, R. P. Intramural Hemorrhage of the Thoracic Aorta. Circulation 1995, 92 (6), 1465-1472.

(14) Cherian, M. P.; Mehta, P.; Kalyanpur, T. M.; Hedgire, S. S.; Narsinghpura, K. S. Arterial interventions in gastrointestinal bleeding. Seminars in Interventional Radiology 2009, 26, 184-195.

(15) Calderon, V. J.; Kasturiarachi, B. M.; Lin, E.; Bansal, V.; Zaidat, O. O. Review of the Mobile Stroke Unit Experience Worldwide. Interventional Neurology 2018, 7 (6), 347-358.
(16) Ay, I.; Blasi, F.; Rietz, T. A.; Rotile, N. J.; Kura, S.; Brownell, A. L.; Day, H.; Oliveira, B. L.; Looby, R. J.; Caravan, P. In Vivo Molecular Imaging of Thrombosis and Thrombolysis Using a FibrinBinding Positron Emission Tomographic Probe. Circulation: Cardiovascular Imaging 2014, 7 (4), 697-705.

(17) Wang, X.; Gkanatsas, Y.; Palasubramaniam, J.; Hohmann, J. D.; Chen, Y. C.; Lim, B.; Hagemeyer, C. E.; Peter, K. Thrombus-Targeted Theranostic Microbubbles: A New Technology towards Concurrent Rapid Ultrasound Diagnosis and Bleeding-free Fibrinolytic Treatment of Thrombosis. Theranostics 2016, 6 (5), 726-38.

(18) Neglén, P.; Raju, S. Intravascular ultrasound scan evaluation of the obstructed vein. Journal of vascular surgery 2002, 35 (4), 694-700.

(19) Rosow, D. E.; Sahani, D.; Strobel, O.; Kalva, S.; MinoKenudson, M.; Holalkere, N. S.; Alsfasser, G.; Saini, S.; Lee, S. I.; Mueller, P. R.; Fernandez-del Castillo, C.; Warshaw, A. L.; Thayer, S. P. Imaging of acute mesenteric ischemia using multidetector CT and CT angiography in a porcine model. J. Gastrointest Surg 2005, 9 (9), $1262-1275$.

(20) Zhang, X.; Bloch, S.; Akers, W.; Achilefu, S. Near-infrared Molecular Probes for In Vivo Imaging. Current Protocols in Cytometry 2012, CHAPTER, 12.27.1-12.27.20.

(21) Marshall, M. V.; Rasmussen, J. C.; Tan, I. C.; Aldrich, M. B.; Adams, K. E.; Wang, X.; Fife, C. E.; Maus, E. A.; Smith, L. A.; SevickMuraca, E. M. Near-Infrared Fluorescence Imaging in Humans with Indocyanine Green: A Review and Update. Open surgical oncology journal (Online) 2010, 2 (2), 12-25.

(22) Colwell, L. J.; Brenner, M. P.; Ribbeck, K. Charge as a selection criterion for translocation through the nuclear pore complex. PLoS Comput. Biol. 2010, 6 (4), e1000747.

(23) Gordon, B. A.; Flanagan, F. L.; Dehdashti, F. Whole-body positron emission tomography: normal variations, pitfalls, and technical considerations. AJR, Am. J. Roentgenol. 1997, 169 (6), $1675-1680$.

(24) Srinivasan, R.; Marchant, R. E.; Gupta, A. S. In vitro and in vivo platelet targeting by cyclic RGD-modified liposomes. J. Biomed. Mater. Res., Part A 2010, 93A (3), 1004-1015.

(25) Ravikumar, M.; Modery, C. L.; Wong, T. L.; Dzuricky, M.; Sen Gupta, A. Mimicking Adhesive Functionalities of Blood Platelets using Ligand-Decorated Liposomes. Bioconjugate Chem. 2012, 23 (6), $1266-1275$

(26) Modery-Pawlowski, C. L.; Tian, L. L.; Ravikumar, M.; Wong, T. L.; Gupta, A. S. In vitro and in vivo hemostatic capabilities of a functionally integrated platelet-mimetic liposomal nanoconstruct. Biomaterials 2013, 34 (12), 3031-3041.

(27) Bertram, J. P.; Williams, C. A.; Robinson, R.; Segal, S. S.; Flynn, N. T.; Lavik, E. B. Intravenous hemostat: nanotechnology to halt bleeding. Sci. Transl. Med. 2009, 1 (11), 11 ra22.

(28) Lashof-Sullivan, M. M.; Shoffstall, E.; Atkins, K. T.; Keane, N.; Bir, C.; VandeVord, P.; Lavik, E. B. Intravenously administered nanoparticles increase survival following blast trauma. Proc. Natl. Acad. Sci. U. S. A. 2014, 111 (28), 10293-10298.

(29) Lashof-Sullivan, M.; Holland, M.; Groynom, R.; Campbell, D.; Shoffstall, A.; Lavik, E. Hemostatic Nanoparticles Improve Survival Following Blunt Trauma Even after 1 Week Incubation at $50{ }^{\circ} \mathrm{C}$. ACS Biomater. Sci. Eng. 2016, 2 (3), 385-392.

(30) Shoffstall, A. J.; Everhart, L. M.; Varley, M. E.; Soehnlen, E. S.; Shick, A. M.; Ustin, J. S.; Lavik, E. B. Tuning Ligand Density on Intravenous Hemostatic Nanoparticles Dramatically Increases Survival Following Blunt Trauma. Biomacromolecules 2013, 14 (8), 27902797.

(31) Shoffstall, A. J.; Atkins, K. T.; Groynom, R. E.; Varley, M. E.; Everhart, L. M.; Lashof-Sullivan, M. M.; Martyn-Dow, B.; Butler, R. S.; Ustin, J. S.; Lavik, E. B. Intravenous hemostatic nanoparticles increase survival following blunt trauma injury. Biomacromolecules 2012, 13 (11), 3850-7.

(32) Shukla, M.; Sekhon, U. D. S.; Betapudi, V.; Li, W.; Hickman, D. A.; Pawlowski, C. L.; Dyer, M. R.; Neal, M. D.; McCrae, K. R.; Sen Gupta, A. In vitro characterization of SynthoPlate (synthetic platelet) 
technology and its in vivo evaluation in severely thrombocytopenic mice. J. Thromb. Haemostasis 2017, 15 (2), 375-387.

(33) Okamura, Y.; Takeoka, S.; Teramura, Y.; Maruyama, H.; Tsuchida, E.; Handa, M.; Ikeda, Y. Hemostatic effects of fibrinogen $\gamma$ chain dodecapeptide-conjugated polymerized albumin particles in vitro and in vivo. Transfusion 2005, 45 (7), 1221-1228.

(34) Hubbard, W. B.; Lashof-Sullivan, M. M.; Lavik, E. B.; VandeVord, P. J. Steroid-Loaded Hemostatic Nanoparticles Combat Lung Injury after Blast Trauma. ACS Macro Lett. 2015, 4 (4), 387391.

(35) Lashof-Sullivan, M.; Shoffstall, A.; Lavik, E. Intravenous hemostats: challenges in translation to patients. Nanoscale 2013, 5 (22), 10719-10728.

(36) Onwukwe, C.; Maisha, N.; Holland, M.; Varley, M.; Groynom, R.; Hickman, D.; Uppal, N.; Shoffstall, A.; Ustin, J.; Lavik, E. Engineering Intravenously Administered Nanoparticles to Reduce Infusion Reaction and Stop Bleeding in a Large Animal Model of Trauma. Bioconjugate Chem. 2018, 29 (7), 2436-2447.

(37) Zolnik, B. S.; Leary, P. E.; Burgess, D. J. Elevated temperature accelerated release testing of PLGA microspheres. J. Controlled Release 2006, 112 (3), 293-300.

(38) Cattaneo, M.; Cerletti, C.; Harrison, P.; Hayward, C. P.; Kenny, D.; Nugent, D.; Nurden, P.; Rao, A. K.; Schmaier, A. H.; Watson, S. P.; Lussana, F.; Pugliano, M. T.; Michelson, A. D. Recommendations for the Standardization of Light Transmission Aggregometry: A Consensus of the Working Party from the Platelet Physiology Subcommittee of SSC/ISTH. J. Thromb. Haemostasis 2013, 11 (6), $1183-1189$.

(39) Kim, D.; El-Shall, H.; Dennis, D.; Morey, T. Interaction of PLGA nanoparticles with human blood constituents. Colloids Surf., B 2005, 40 (2), 83-91.

(40) Watson, P. M.; Humphries, M. J.; Relton, J.; Rothwell, N. J.; Verkhratsky, A.; Gibson, R. M. Integrin-binding RGD peptides induce rapid intracellular calcium increases and MAPK signaling in cortical neurons. Mol. Cell. Neurosci. 2007, 34 (2), 147-54.

(41) Vavrusova, M.; Skibsted, L. H. Calcium binding to dipeptides of aspartate and glutamate in comparison with orthophosphoserine. J. Agric. Food Chem. 2013, 61 (22), 5380-4.

(42) Lee, H. B.; Blaufox, M. D. Blood volume in the rat. J. Nucl. Med. 1985, 26 (1), 72-76.

(43) Blanco, E.; Shen, H.; Ferrari, M. Principles of nanoparticle design for overcoming biological barriers to drug delivery. Nat. Biotechnol. 2015, 33 (9), 941-951.

(44) Wiedeman, M. P. Dimensions of blood vessels from distributing artery to collecting vein. Circ. Res. 1963, 12, 375-8.

(45) van der Meer, P. F.; Tomson, B.; Brand, A. In vivo tracking of transfused platelets for recovery and survival studies: an appraisal of labeling methods. Transfus. Apher. Sci. 2010, 42 (1), 53-61.

(46) Salem, A. K.; Cannizzaro, S. M.; Davies, M. C.; Tendler, S. J.; Roberts, C. J.; Williams, P. M.; Shakesheff, K. M. Synthesis and characterisation of a degradable poly(lactic acid)-poly(ethylene glycol) copolymer with biotinylated end groups. Biomacromolecules 2001, 2 (2), 575-80.

(47) Daniel, M. C.; Astruc, D. Gold nanoparticles: assembly, supramolecular chemistry, quantum-size-related properties, and applications toward biology, catalysis, and nanotechnology. Chem. Rev. (Washington, DC, U. S.) 2004, 104 (1), 293-346.

(48) Gkikas, M.; Timonen, J.; Ruokolainen, J.; Alexandridis, P.; Iatrou, H. Facile aqueous synthesis and stabilization of nearly monodispersed gold nanospheres by poly(L-proline). J. Polym. Sci., Part A: Polym. Chem. 2013, 51 (6), 1448-1456.

(49) Bhattacharya, R.; Mukherjee, P. Biological properties of "naked" metal nanoparticles. Adv. Drug Delivery Rev. 2008, 60 (11), 1289-306.

(50) Connor, E. E.; Mwamuka, J.; Gole, A.; Murphy, C. J.; Wyatt, M. D. Gold nanoparticles are taken up by human cells but do not cause acute cytotoxicity. Small 2005, 1 (3), 325-7.
(51) De, M.; Ghosh, P. S.; Rotello, V. M. Applications of Nanoparticles in Biology. Adv. Mater. (Weinheim, Ger.) 2008, 20 (22), 4225-4241.

(52) Hainfeld, J. F.; Slatkin, D. N.; Focella, T. M.; Smilowitz, H. M. Gold nanoparticles: a new X-ray contrast agent. Br. J. Radiol. 2006, 79 (939), 248-53.

(53) Brust, M.; Walker, M.; Bethell, D.; Schiffrin, D. J.; Whyman, R. Synthesis of thiol-derivatised gold nanoparticles in a two-phase Liquid-Liquid system. J. Chem. Soc., Chem. Commun. 1994, O (7), 801-802.

(54) Clark, D. P.; Ghaghada, K.; Moding, E. J.; Kirsch, D. G.; Badea, C. T. In vivo characterization of tumor vasculature using iodine and gold nanoparticles and dual energy micro-CT. Phys. Med. Biol. 2013, 58 (6), 1683-704.

(55) Hainfeld, J. F.; O'Connor, M. J.; Dilmanian, F. A.; Slatkin, D. N.; Adams, D. J.; Smilowitz, H. M. Micro-CT enables microlocalisation and quantification of Her2-targeted gold nanoparticles within tumour regions. Br. J. Radiol. 2011, 84 (1002), 526-33.

(56) Vijayakumar, C.; Balan, B.; Saeki, A.; Tsuda, T.; Kuwabata, S.; Seki, S. Gold Nanoparticle Assisted Self-Assembly and Enhancement of Charge Carrier Mobilities of a Conjugated Polymer. J. Phys. Chem. C 2012, 116 (33), 17343-17350.

(57) Ou, Y.-Y.; Huang, M. H. High-Density Assembly of Gold Nanoparticles on Multiwalled Carbon Nanotubes Using 1-Pyrenemethylamine as Interlinker. J. Phys. Chem. B 2006, 110 (5), 20312036.

(58) De Jong, W. H.; Hagens, W. I.; Krystek, P.; Burger, M. C.; Sips, A. J.; Geertsma, R. E. Particle size-dependent organ distribution of gold nanoparticles after intravenous administration. Biomaterials 2008, 29 (12), 1912-9. 